\title{
Spinor vertices and bubbles in the old conformal bootstrap in AdS/CFT and the fermion mass hierarchy problem: The Yukawa model
}

\author{
Boris L. Altshuler $\odot^{*}$ \\ Theoretical Physics Department, P.N. Lebedev Physical Institute, \\ 53 Leninsky Prospect, Moscow 119991, Russia
}

(Received 19 November 2019; accepted 19 January 2020; published 19 February 2020)

\begin{abstract}
Attempts to resolve the long-standing Fermion mass hierarchy problem in frames of the AdS/CFT correspondence demand the knowledge of bulk fermion masses. The approach of the "old" conformal bootstrap in the anti-de Sitter (AdS) context permitted calculating bulk masses of scalar fields, as it was shown in three previous papers B. L. Altshuler [arXiv:1810.01105; Int. J. Mod. Phys. 2050001 (2020); J. High Energy Phys. 01 (2020) 137]. In the present paper, this approach is extended to physically more interesting spin-1/2 bulk fields. Calculation of spinor-scalar vertices is performed in physical AdS space, and unexpectedly simple expressions for spinor-scalar bubbles (two-point one-loop self-energy Witten diagrams) are obtained. The "double-trace from UV to IR flow" subtraction of UV divergences used earlier in calculations of the UV-finite bulk tadpoles is applied to bubbles. This permitted us to write down in the frame of the old conformal bootstrap approach the nontrivial spectral equations for the bulk fermion masses; the SU(N) Yukawa model of spinor fields interacting with conformal invariant scalar field in case of four boundary dimensions is considered as a specific example.
\end{abstract}

DOI: 10.1103/PhysRevD.101.046021

\section{INTRODUCTION}

Explaining the fermion mass hierarchy (also called the flavor hierarchy problem) remains a challenge in theoretical physics (see, e.g., Refs. [1,2] and references therein). I cannot help but quote the very beginning of Ref. [2]: "In a recent interview published in CERN COURIER, Steven Weinberg was asked what single open question he would like to see answered in his lifetime, and Weinberg replied that it is only the mystery of the observed pattern of quarks and leptons masses [3]".

In frames of the AdS/CFT approach and two-branes Randall-Sundrum model [4] (Poincaré coordinate $\epsilon<z<$ $L$ where $\epsilon^{-1} \sim M_{\mathrm{Pl}}=10^{19} \mathrm{GeV}$ for the Planck scale and $L^{-1} \sim M_{\mathrm{EW}}=10^{2} \mathrm{GeV}$ for the electroweak scale), spectra of physical particles (glueballs, mesons, light and heavy fermions, etc.) are obtained as eigenvalues of equations for bulk fields, and it is possible in principle to get the lookedfor fermion masses of intermediate scales with the choice of dynamics in the bulk and of boundary conditions on both branes [5-9]. It is evident that there is great arbitrariness in this approach.

\footnotetext{
*baltshuler@yandex.ru, altshul@1pi.ru
}

Published by the American Physical Society under the terms of the Creative Commons Attribution 4.0 International license. Further distribution of this work must maintain attribution to the author(s) and the published article's title, journal citation, and DOI. Funded by SCOAP ${ }^{3}$.
Here, I mention one of possibilities to overcome this arbitrariness. This possibility is a rather natural "twisted" boundary condition for spin $1 / 2$ fields that permits to get observable fermion masses of any scale depending only on the bulk masses of Fermi fields in higher dimensions $[10,11]$. The spectral equation in this case looks like (see formula (29) in Ref. [11])

$$
\frac{J_{\alpha-1}\left(m_{n} \epsilon\right)}{Y_{\alpha-1}\left(m_{n} \epsilon\right)}=\frac{J_{\alpha}\left(m_{n} L\right)}{Y_{\alpha}\left(m_{n} L\right)},
$$

where physical four-momentum $p_{n}^{2}=-m_{n}^{2}, J_{\alpha}, Y_{\alpha}$ are Bessel functions of the first and second kinds of order $\alpha=$ $m / k_{\text {AdS }}+1 / 2$ [ $m$ is bulk mass of fermion field, and $k_{\text {AdS }}$ is anti-de Sitter (AdS) space curvature], values of $\epsilon$ and $L$ are given above. Surely, Eq. (1) has a tower of solutions beginning from the electroweak scale $L^{-1}$. But it also has a special solution when both arguments of Bessel functions in (1) are small:

$$
p_{0}=\frac{1}{L}\left(\frac{\epsilon}{L}\right)^{\alpha-1} \sqrt{4 \alpha(\alpha-1)} .
$$

It is seen that for $1<\alpha<2$, that is for

$$
\frac{1}{2}<\frac{m}{k_{\mathrm{AdS}}}<\frac{3}{2},
$$

$p_{0}$ may have any value in the interval from the electroweak scale $M_{\mathrm{EW}}$ to the electron neutrino (or gravitino) mass scale 
of order $M_{\mathrm{EW}}^{2} / M_{\mathrm{Pl}}$. Thus, well-grounded calculations of fermions bulk masses may open the way to the solution of the Fermion mass hierarchy problem. This was the main motivation of the present paper.

One of the candidates for the theory capable of fixing masses of bulk fields may be the "old" conformal bootstrap in the AdS/CFT context $[12,13]$. The old conformal bootstrap was proposed about 50 years ago in pioneer papers [14,15] and developed in Refs. [16-21] (see, e.g., Ref. [22] and references therein) as a non-Lagrangian tool of self-consistent calculations of conformal dimensions.

In the AdS/CFT context, finding conformal dimensions is equivalent to finding bulk masses. In Ref. [12], the values of bulk masses of scalar fields that are the roots of corresponding bootstrap spectral equations were found in certain models under the oversimplifying assumption of replacing of two "intermediate" Green's functions in selfenergy Witten diagrams (bubbles) by their harmonic counterparts. In Ref. [13], this assumption was abandoned, and UV divergence of the bubble was subtracted in the "double-trace from UV to IR flow" way used earlier in calculations of the UV-finite tadpoles and bulk vacuum quantum energies of scalar [23-28] and spinor [29,30] bulk fields. The sensible, that is, obeying the unitarity bound demand, values of conformal dimensions of scalar fields in the $O(N)$ symmetric model were obtained in Ref. [13] for $N=1 \ldots 4$ in the case of $d=4$ boundary dimensions.

To extend the approach of Ref. [13] to physically more interesting spin-1/2 bulk fields is the goal of the present paper.

The Dirac field of spin $1 / 2$ was studied sufficiently well from the AdS/CFT perspective-see earlier papers [29] and [31-35], in which in particular spinor bulk-to-bulk and bulk-to-boundary propagators on AdS were written down, and recent works [36-39]. In Refs. [37,38] spinor-spinorscalar vertices were calculated in the formalism of embedding space, and also in Ref. [38], the spectral representation of the bulk spinor Green's function is presented. The bulk fermion loop of the scalar field was first calculated in Ref. [39] also in the formalism of embedding space, whereas the one-loop self-energy of the Fermi field on AdS was not calculated earlier, as to my knowledge. Here, I do not use the formalism of embedding space and perform calculations in physical $\mathrm{AdS}_{d+1}$.

In Sec. II, some well-known expressions are presented and include four bulk-to-boundary propagators, the spectral representation of Green's function, and the split representation of the harmonic function for the spin- $1 / 2$ bulk field. Also, Sec. II presents novel bulk and conformal integrals necessary for the calculation of spinor-scalar vertices and bubbles; their derivation is given in the Appendix.

In Sec. III, calculation of spinor-spinor-scalar three-point correlators (vertices) is performed. There are two types of such correlators: type I in which two spinor fields are of one and the same asymptotic at the AdS horizon (symbolically, those are correlators IR $-\mathrm{IR}-\phi$ or UV $-\mathrm{UV}-\phi$, where $\phi$ is a scalar field), and type II which are three-point correlators IR $-\mathrm{UV}-\phi$ or UV $-\mathrm{IR}-\phi$. The first type of these correlators is well known and was extensively used in calculations of spinor-scalar Witten diagrams [34-39], whereas vertices of the second type have not been written down so far, as to my knowledge.

In Sec. IV, the one-loop quantum contributions (bubbles) to the two-point boundary-to-boundary conformal correlators of the scalar field (the loop is formed by two spinor bulk Green's functions) and of the spinor field (the loop is formed by spinor and scalar Green's functions) are calculated. The double integral spectral representations are given for both bubbles, repeating the approach of Ref. [40] in which double integral spectral representations were put down for bubbles formed by the fields of integer spin. The numerators of integrands in these representations are formed like ordinary bubbles but with the replacement in their expressions of intermediate Green's functions to the corresponding harmonic functions (proportional to the difference of "UV" and "IR" Green's functions). Calculations of harmonic bubbles formed by two spinors and by a spinor and scalar are the main contents of Sec. IV. The reward for these rather lengthy calculations is the simplicity of the final formulas for both bubbles expressed through one and the same universal function of conformal dimensions; moreover: for an even number $d$ of boundary dimensions, this universal function is just a combination of elementary functions.

In Sec. V, UV divergence of the bubbles is subtracted with a tool used earlier in calculations of the UV-finite Witten tadpoles [23-30]. The tool is simple, it says that, instead of ordinary Witten diagrams, the difference of two similar Witten diagrams built of UV and IR bulk Green's functions must be considered. This difference is the deformation in amplitudes under double-trace flow from UV to IR boundary conditions, as was first proposed in Ref. [41] (see Ref. [42] and references therein). A general concept is put forward for such a crucial redefinition of quantum amplitudes: it is proposed to construct Witten diagrams using the quantum generation functional (68), which is the ratio of two standard functionals built with use of UV and IR boundary conditions; most generally, this approach is studied in Ref. [43], in which it was shown that in "ratio functionals" functional integrals over fields' bulk degrees of freedom reduce, and only quantum theory of boundary fields is taken into account. It is shown in the paper that this procedure gives well-defined UV-finite expressions for bubble diagrams, but whether it will work for triangle and other Witten diagrams is an open question.

In Sec. VI, transparent formulas for two spinors and spinor-scalar bubbles are derived in the $S U(N)$ model with Yukawa interaction of $N$ spinor fields with the conformal invariant scalar field, in the case of four boundary dimensions. 
In Sec. VII, results of Sec. VI are used to write down the old conformal bootstrap spectral equations for bulk fermion mass $m$ in the $S U(N)$ symmetric option when masses of all $N$ spinors are equal. The system of $N$ spectral equations that will permit us to study the possibility of the spontaneous breakdown of $S U(N)$ symmetry is also presented in Sec. VII.

The Conclusion sums up three principle results of the paper and outlines the possible directions of future work.

\section{PRELIMINARIES}

\section{A. Scalar field on AdS}

We work in Euclidean $\mathrm{AdS}_{d+1}$ in Poincaré coordinates $Z^{\mu}=\left\{z_{0}, \vec{z}\right\}(\mu=0,1, \ldots d)$, where AdS curvature $k_{\text {AdS }}$ is put equal to 1 ,

$$
d s^{2}=\frac{d z_{0}^{2}+d \vec{z}^{2}}{z_{0}^{2}}
$$

and consider bulk scalar and spinor fields.

Bulk scalar field $\phi(X)$ of mass $M$ is dual to the boundary conformal operator $O_{\Delta_{\phi}}^{\mathrm{IR}}(\vec{x})$ or to its "shadow" operator $O_{d-\Delta_{\phi}}^{\mathrm{UV}}(\vec{x})$ with scaling dimensions

$\Delta_{\phi}^{\mathrm{IR}} \equiv \Delta_{\phi}=\frac{d}{2}+\nu ; \quad \nu=\sqrt{\frac{d^{2}}{4}+M^{2}} ; \quad \Delta_{\phi}^{\mathrm{UV}}=d-\Delta_{\phi}$.

We take the normalization of the scalar field's bulk-toboundary propagator and of the corresponding conformal correlator like in Ref. [40]:

$$
\begin{aligned}
K_{\Delta}(Z, \vec{x}) & =\lim _{x_{0} \rightarrow 0}\left[\frac{G_{\Delta}^{B B}(Z, X)}{\left(x_{0}\right)^{\Delta}}\right]=C_{\Delta} \cdot Q^{\Delta}(Z, \vec{x}), \\
C_{\Delta} & =\frac{\Gamma(\Delta)}{2 \pi^{d / 2} \Gamma\left(1+\Delta-\frac{d}{2}\right)}, \quad Q(Z, \vec{x})=\frac{z_{0}}{z_{0}^{2}+(\vec{z}-\vec{x})^{2}}
\end{aligned}
$$

and

$$
\left\langle O_{\Delta}(\vec{x}) O_{\Delta}(\vec{y})\right\rangle=\lim _{\substack{x_{0} \rightarrow 0 \\ y_{0} \rightarrow 0}}\left[\frac{G_{\Delta}^{B B}(X, Y)}{\left(x_{0} y_{0}\right)^{\Delta}}\right]=\frac{C_{\Delta}}{P_{x y}^{\Delta}}, P_{x y}=|\vec{x}-\vec{y}|^{2} .
$$

The bulk-to-bulk IR $\left[\Delta=\Delta^{\mathrm{IR}}>d / 2\right.$, see Eq. (5)] scalar field Green's function $G_{\Delta}^{\mathrm{IR}}(X, Y)$ possesses the KallenLehmann-type spectral representation in which the numerator of the integrand (harmonic function) admits split representation $[40,44-47]$ :

$$
\begin{aligned}
G_{\Delta}^{\mathrm{IR}}(X, Y) & =\int_{-\infty}^{+\infty} \frac{\Omega_{c, 0}(X, Y) d c}{\left[c^{2}+\left(\Delta-\frac{d}{2}\right)^{2}\right]}, \\
\Omega_{c, 0}(X, Y) & =\frac{c^{2}}{\pi} \int K_{\frac{d}{2}+i c}\left(X, \vec{x}_{a}\right) K_{\frac{d}{2}-i c}\left(Y, \vec{x}_{a}\right) d^{d} \vec{x}_{a},
\end{aligned}
$$

where

$$
\Omega_{c, 0}(X, Y)=\frac{i c}{2 \pi} \tilde{G}_{\frac{d}{2}+i c}
$$

is a scalar field harmonic function which is proportional to the difference (marked here with a tilde) of IR and UV bulk Green's functions:

$$
\begin{aligned}
\tilde{G}_{\Delta}(X, Y) & =G_{\Delta}^{\mathrm{IR}}-G_{d-\Delta}^{\mathrm{UV}} \\
& =(d-2 \Delta) \int K_{\Delta}\left(X, \vec{x}_{a}\right) K_{d-\Delta}\left(Y, \vec{x}_{a}\right) d^{d} \vec{x}_{a} .
\end{aligned}
$$

\section{B. Spinor field on AdS}

Bulk spinor field $\psi(X)$ of mass $m$ on $\operatorname{AdS}_{d+1}$ obeys the Dirac equation [31-39]

$$
\begin{aligned}
\left(\gamma^{\mu} D_{\mu}-m\right) \psi(X) & =\left(z_{0} \gamma^{0} \frac{\partial}{\partial z_{0}}+z_{0} \vec{\gamma} \frac{\partial}{\partial \vec{z}}-\frac{d}{2} \gamma^{0}-m\right) \psi(X) \\
& =0
\end{aligned}
$$

where $\gamma^{\mu}=\left\{\gamma^{0}, \vec{\gamma}\right\}$ are standard anticommuting gamma matrices in $(d+1)$-dimensional Euclidean space: $\gamma^{\mu} \gamma^{\nu}+$ $\gamma^{\nu} \gamma^{\mu}=2 \delta^{\mu \nu}$. The bulk spinor field of mass $m$ is dual to boundary conformal operator $O_{\Delta_{\psi}}^{\mathrm{IR}}(\vec{x})$ or to its "shadow" operator $O_{d-\Delta_{\psi}}^{\mathrm{UV}}(\vec{x})$ with scaling dimensions

$$
\begin{aligned}
\Delta_{\psi}^{\mathrm{IR}} & =\frac{d}{2}+m+\frac{1}{2} \equiv \Delta_{\psi}+\frac{1}{2} \\
\Delta_{\psi}^{\mathrm{UV}} & =\frac{d}{2}-m+\frac{1}{2}=d-\Delta_{\psi}+\frac{1}{2} .
\end{aligned}
$$

Corresponding conformal correlators are

$$
\begin{aligned}
\left\langle\bar{O}_{\Delta_{\psi}}^{\mathrm{IR}}(\vec{x}) O_{\Delta_{\psi}}^{\mathrm{IR}}(\vec{y})\right\rangle & =\hat{C}_{\Delta_{\psi}} \frac{\vec{\gamma}(\vec{x}-\vec{y}) \Pi_{-}}{P_{x y}^{\Delta_{\psi}+\frac{1}{2}}}, \\
\hat{C}_{\Delta_{\psi}} & =\frac{\Gamma\left(\Delta_{\psi}+\frac{1}{2}\right)}{\pi^{d / 2} \Gamma\left(\Delta_{\psi}+\frac{1}{2}-\frac{d}{2}\right)}, \\
\left\langle\bar{O}_{d-\Delta_{\psi}}^{\mathrm{UV}}(\vec{x}) O_{d-\Delta_{\psi}}^{\mathrm{UV}}(\vec{y})\right\rangle & =\hat{C}_{d-\Delta_{\psi}} \frac{\vec{\gamma}(\vec{x}-\vec{y}) \Pi_{+}}{P_{x y}^{d-\Delta_{\psi}+\frac{1}{2}}},
\end{aligned}
$$

where $\Pi_{ \pm}$are projective operators: 


$$
\Pi_{ \pm}=\frac{1}{2}\left(1 \pm \gamma^{0}\right), \quad \Pi_{+}^{2}=\Pi_{+}, \quad \Pi_{-}^{2}=\Pi_{-},
$$

$\Pi_{+} \Pi_{-}=0, \quad \Pi_{+} \vec{\gamma}=\vec{\gamma} \Pi_{-}$.

Bulk spinor Green's functions $S^{\mathrm{IR}, \mathrm{UV}}(X, Y)$ that possess IR or UV asymptotic at the horizon, $x_{0}, y_{0} \rightarrow 0$, and that are zero at the AdS infinity, $x_{0}, y_{0} \rightarrow \infty$, are well known; see, e.g., in Refs. [29,34,35]. Their properly normalized limits at the horizon give four spinor bulk-to-boundary propagators $\Sigma^{\mathrm{IR}(\mathrm{UV})}$ and $\bar{\Sigma}^{\mathrm{IR}(\mathrm{UV})}$, which permit expressing bulk solutions of Dirac equation (11) $\psi(Z), \bar{\psi}(Z)$ through the boundary fields [31-35]. Those bulk-to-boundary propagators are the main tool in calculations of this paper,

$$
\begin{aligned}
\Sigma_{\Delta_{\psi}}^{\mathrm{IR}}(Z, \vec{y}) & =\lim _{y_{0} \rightarrow 0}\left[\frac{S_{\Delta_{\psi}}^{\mathrm{IR}}(Z, Y)}{y_{0}^{\Delta_{\psi}+\frac{1}{2}}}\right] \\
& =\hat{C}_{\Delta_{\psi}} Q^{\Delta_{\psi}+\frac{1}{2}}(Z, \vec{y}) \frac{\left[z_{0}-\vec{\gamma}(\vec{z}-\vec{y})\right]}{\sqrt{z_{0}}} \Pi_{-}, \\
\bar{\Sigma}_{\Delta_{\psi}}^{\mathrm{IR}}(Z, \vec{x}) & =\lim _{x_{0} \rightarrow 0}\left[\frac{S_{\Delta_{\psi}}^{\mathrm{IR}}(X, Z)}{x_{0}^{\Delta_{\psi}+\frac{1}{2}}}\right] \\
& =\hat{C}_{\Delta_{\psi}} Q^{\Delta_{\psi}+\frac{1}{2}}(Z, \vec{x}) \Pi_{+} \frac{\left[z_{0}+\vec{\gamma}(\vec{z}-\vec{x})\right]}{\sqrt{z_{0}}} .
\end{aligned}
$$

and in a similar way for $\Sigma^{\mathrm{UV}}$ and $\bar{\Sigma}^{\mathrm{UV}}$,

$$
\Sigma_{d-\Delta_{\psi}}^{\mathrm{UV}}(Z, \vec{y})=\hat{C}_{d-\Delta_{\psi}} Q^{d-\Delta_{\psi}+\frac{1}{2}}(Z, \vec{y}) \frac{\left[z_{0}+\vec{\gamma}(\vec{z}-\vec{y})\right]}{\sqrt{z_{0}}} \Pi_{+},
$$

$\bar{\Sigma}_{d-\Delta_{\psi}}^{\mathrm{UV}}(Z, \vec{x})=\hat{C}_{d-\Delta_{\psi}} Q^{d-\Delta_{\psi}+\frac{1}{2}}(Z, \vec{x}) \Pi_{-} \frac{\left[z_{0}-\vec{\gamma}(\vec{z}-\vec{x})\right]}{\sqrt{z_{0}}}$,

where $\hat{C}_{\Delta_{\psi}}$ and $Q(Z, \vec{x})$ are defined correspondingly in (13) and (6).

Conformal spinor correlators (13) are obtained from (15)-(18) when the bulk coordinate is sent to horizon in these expressions.

Analogous to the scalar case (8), the spectral representation for $S_{\Delta_{\psi}}^{\mathrm{IR}}(X, Y)\left[\Delta_{\psi}=d / 2+m>d / 2\right.$; see Eq. (12)] was given in Ref. [38],

$$
\begin{aligned}
S_{\Delta_{\psi}}^{\mathrm{IR}}(X, Y) & =\int_{-\infty}^{+\infty} \frac{\Omega_{c, 1 / 2}(X, Y) d c}{\left[c+i\left(\Delta_{\psi}-\frac{d}{2}\right)\right]}, \\
\Omega_{c, 1 / 2}(X, Y) & =\frac{i}{2 \pi} \tilde{S}_{\Delta_{c}}(X, Y), \quad \Delta_{c}=\frac{d}{2}+i c,
\end{aligned}
$$

where spinor harmonic function $\Omega_{c, 1 / 2}(X, Y)$ is proportional to the difference (again marked by a tilde) of IR and UV Green's functions, that possesses nice split representations through bulk-to-boundary propagators (15)-(18) integrated over common boundary point [38] [cf. (9) and (10) for the scalar field case]:

$$
\begin{aligned}
\tilde{S}_{\Delta_{\psi}}(X, Y) & =S_{\Delta_{\psi}}^{\mathrm{IR}}(X, Y)-S_{d-\Delta_{\psi}}^{\mathrm{UV}}(X, Y) \\
& =\int \Sigma_{\Delta_{\psi}}^{\mathrm{IR}}\left(X, \vec{x}_{a}\right) \bar{\Sigma}_{d-\Delta_{\psi}}^{\mathrm{UV}}\left(Y, \vec{x}_{a}\right) d \vec{x}_{a} \\
& =\int \Sigma_{d-\Delta_{\psi}}^{\mathrm{UV}}\left(X, \vec{x}_{a}\right) \bar{\Sigma}_{\Delta_{\psi}}^{\mathrm{IR}}\left(Y, \vec{x}_{a}\right) d \vec{x}_{a} .
\end{aligned}
$$

\section{Some integrals}

We shall need two following bulk integrals for two values of parameter $(\alpha: 0$ and $\alpha: 1)$ :

$$
\begin{aligned}
& \mathrm{D}_{\gamma_{1}, \gamma_{2}, \gamma_{3}}^{(\alpha)}\left(\vec{x}_{1}, \vec{x}_{2}, \vec{x}_{3}\right) \\
& \quad=\int Q^{\gamma_{1}}\left(Z, \vec{x}_{1}\right) Q^{\gamma_{2}}\left(Z, \vec{x}_{2}\right) Q^{\gamma_{3}}\left(Z, \vec{x}_{3}\right) \frac{d Z}{z_{0}^{\alpha}},
\end{aligned}
$$

$Q(Z, \vec{x})$ see in (6). $\mathrm{D}^{(0)}$ is well known; it gives the threepoint scalar fields vertex $[44,48,49]$,

$$
\begin{aligned}
\mathrm{D}_{\gamma_{1}, \gamma_{2}, \gamma_{3}}^{(0)}\left(\vec{x}_{1}, \vec{x}_{2}, \vec{x}_{3}\right) & =\frac{\mathrm{d}^{(0)}\left(\gamma_{1}, \gamma_{2}, \gamma_{3}\right)}{P_{12}^{\delta_{12}} P_{13}^{\delta_{13}} P_{23}^{\delta_{23}}}, \\
\mathrm{~d}^{(0)}\left(\gamma_{1}, \gamma_{2}, \gamma_{3}\right) & =\frac{\pi^{d / 2} \Gamma \frac{\Gamma\left(\frac{\Sigma \gamma_{i}-d}{2}\right) \Gamma\left(\delta_{12}\right) \Gamma\left(\delta_{13}\right) \Gamma\left(\delta_{23}\right)}{2},}{\Gamma\left(\gamma_{1}\right) \Gamma\left(\gamma_{2}\right) \Gamma\left(\gamma_{3}\right)},
\end{aligned}
$$

where

$$
\begin{aligned}
& \delta_{12}=\frac{\gamma_{1}+\gamma_{2}-\gamma_{3}}{2} ; \quad \delta_{13}=\frac{\gamma_{1}+\gamma_{3}-\gamma_{2}}{2} ; \\
& \delta_{23}=\frac{\gamma_{2}+\gamma_{3}-\gamma_{1}}{2}
\end{aligned}
$$

Whereas $\mathrm{D}^{(1)}$ is derived in Appendix, it will be used in the calculation of the spinor-spinor-scalar vertex of type II in Sec. III. B:

$$
\begin{aligned}
\mathrm{D}_{\gamma_{1}, \gamma_{2}, \gamma_{3}}^{(1)}\left(\vec{x}_{1}, \vec{x}_{2}, \vec{x}_{3}\right)= & \frac{\mathrm{d}^{(1)}\left(\gamma_{1}, \gamma_{2}, \gamma_{3}\right)}{P_{12}^{\hat{\delta}_{12}} P_{13}^{\hat{\delta}_{13}} \hat{\delta}_{23}} \\
& \times\left[\frac{\hat{\delta}_{12} \hat{\delta}_{13}}{P_{12} P_{13}}+\frac{\hat{\delta}_{12} \hat{\delta}_{23}}{P_{12} P_{23}}+\frac{\hat{\delta}_{13} \hat{\delta}_{23}}{P_{13} P_{23}}\right] \\
\mathrm{d}^{(1)}\left(\gamma_{1}, \gamma_{2}, \gamma_{3}\right)= & \frac{\pi^{d / 2}}{2} \frac{\Gamma\left(\frac{\Sigma \gamma_{i}-d-1}{2}\right) \Gamma\left(\hat{\delta}_{12}\right) \Gamma\left(\hat{\delta}_{13}\right) \Gamma\left(\hat{\delta}_{23}\right)}{\Gamma\left(\gamma_{1}\right) \Gamma\left(\gamma_{2}\right) \Gamma\left(\gamma_{3}\right)} \\
\hat{\delta}_{i j} & =\delta_{i j}-\frac{1}{2} .
\end{aligned}
$$

There are relations between coefficients $\mathrm{d}^{(0)}$ and $\mathrm{d}^{(1)}$ that permit significantly simplifying the calculations, 


$$
\mathrm{d}^{(0)}\left(\gamma_{1}-1, \gamma_{2}, \gamma_{3}\right)=\mathrm{d}^{(1)}\left(\gamma_{1}, \gamma_{2}, \gamma_{3}\right)\left(\gamma_{1}-1\right) \hat{\delta}_{23},
$$

and the same for two other arguments.

Along with the well-known conformal integrals $[16,17,50]$,

$R_{\beta_{1} \beta_{2} \beta_{3}}^{(0)}\left(\vec{x}_{1}, \vec{x}_{2}, \vec{x}_{3}\right)=\int \frac{d^{d} \vec{y}}{P_{1 y}^{\beta_{1}} P_{2 y}^{\beta_{2}} P_{3 y}^{\beta_{3}}} \stackrel{\Sigma \beta_{i}=d}{=} \frac{A\left(\beta_{1}, \beta_{2}, \beta_{3}\right)}{P_{12}^{\frac{d}{2}-\beta_{3}} P_{13}^{\frac{d}{2}-\beta_{2}} P_{23}^{\frac{d}{2}-\beta_{1}}}$,

and

$$
\int \frac{d^{d} \vec{y}}{P_{1 y}^{\beta_{1}} P_{2 y}^{\beta_{2}}}=\frac{A\left(\beta_{1}, \beta_{2}, d-\beta_{1}-\beta_{2}\right)}{P_{12}^{\beta_{1}+\beta_{2}-\frac{d}{2}}},
$$

where

$$
A\left(\beta_{1}, \beta_{2}, \beta_{3}\right)=\frac{\pi^{d / 2} \Gamma\left(\frac{d}{2}-\beta_{1}\right) \Gamma\left(\frac{d}{2}-\beta_{2}\right) \Gamma\left(\frac{d}{2}-\beta_{3}\right)}{\Gamma\left(\beta_{1}\right) \Gamma\left(\beta_{2}\right) \Gamma\left(\beta_{3}\right)},
$$

the knowledge of two following integrals is necessary for calculation of spinor one-loop self-energy in Sec. IV. B:

$$
\begin{aligned}
R_{\beta_{1} \beta_{2} \beta_{3}}^{(1)}\left(\vec{x}_{1}, \vec{x}_{2}, \vec{x}_{3}\right)= & \int \frac{d^{d} \vec{y}}{P_{1 y}^{\beta_{1}} P_{2 y}^{\beta_{2}} P_{3 y}^{\beta_{3}}} \stackrel{\sum \beta_{i}=d+1}{=} \frac{A\left(\beta_{1}, \beta_{2}, \beta_{3}\right)}{P_{12}^{\frac{d}{2}-\beta_{3}} P_{13}^{\frac{d}{2}-\beta_{2}} P_{23}^{\frac{d}{2}-\beta_{1}}} \\
& \cdot\left[\frac{\left(\frac{d}{2}-\beta_{2}\right)\left(\frac{d}{2}-\beta_{3}\right)}{P_{12} P_{13}}+\frac{\left(\frac{d}{2}-\beta_{1}\right)\left(\frac{d}{2}-\beta_{2}\right)}{P_{13} P_{23}}\right. \\
& \left.+\frac{\left(\frac{d}{2}-\beta_{1}\right)\left(\frac{d}{2}-\beta_{3}\right)}{P_{12} P_{23}}\right],
\end{aligned}
$$

and

$$
\begin{aligned}
\int d \vec{x}_{a} \frac{\vec{\gamma}\left(\vec{x}_{2}-\vec{x}_{a}\right)}{P_{1 a}^{\beta_{1}} P_{2 a}^{\beta_{2}}}= & \frac{\vec{\gamma}\left(\vec{x}_{1}-\vec{x}_{2}\right)}{P_{12}^{\beta_{1}+\beta_{2}-\frac{d}{2}}} \\
& \cdot \frac{1}{2}\left[A\left(\beta_{1}-1, \beta_{2}, d-\beta_{1}-\beta_{2}+1\right)\right. \\
& -A\left(\beta_{1}, \beta_{2}-1, d-\beta_{1}-\beta_{2}+1\right) \\
& \left.-A\left(\beta_{1}, \beta_{2}, d-\beta_{1}-\beta_{2}\right)\right] .
\end{aligned}
$$

The derivation of (29) is given in the Appendix, whereas expression (30) is easy to prove by multiplying it by $\vec{\gamma}\left(\vec{x}_{1}-\right.$ $\vec{x}_{2}$ ) with account of (27) and the identity

$$
\begin{aligned}
\vec{\gamma}\left(\vec{x}_{1}-\vec{x}_{2}\right) \cdot \vec{\gamma}\left(\vec{x}_{2}-\vec{x}_{a}\right)= & \frac{1}{2}\left[P_{1 a}-P_{2 a}-P_{12}\right] \\
& +\left(\vec{x}_{1}-\vec{x}_{2}\right)^{\alpha}\left(\vec{x}_{2}-\vec{x}_{a}\right)^{\beta} S^{\alpha \beta}, \\
S^{\alpha \beta}= & \frac{\gamma^{\alpha} \gamma^{\beta}-\gamma^{\beta} \gamma^{\alpha}}{2} .
\end{aligned}
$$

Because of the $\alpha \leftrightarrow \beta$ asymmetry of the $S^{\alpha \beta}$ term in (31), its contribution to the rhs of (30) is zero.

\section{SPINOR-SPINOR-SCALAR VERTICES}

\section{A. Spinor-spinor-scalar vertex: Type I}

Three-point correlators of scalar field $\phi$ of conformal dimension $\Delta_{\phi}$ and of two spinor fields $\psi_{1}$ and $\psi_{2}$, generally speaking of different conformal dimensions $\Delta_{\psi_{1}}^{\mathrm{IR}(\mathrm{UV})}$ and $\Delta_{\psi_{2}}^{\mathrm{IR}(\mathrm{UV})}$ [see (12)] are generated by the bulk Yukawa interaction $\mathcal{L}_{\text {int }}=g \cdot \bar{\psi}_{1}(Z) \psi_{2}(Z) \phi(Z)$.

These three-point correlators are of two essentially different types, with regard to spinor fields: (I) of coinciding, $\overline{\mathrm{IR}}-\mathrm{IR}$ or $\overline{\mathrm{UV}}-\mathrm{UV}$, and (II) of the opposite, $\overline{\mathrm{IR}}-$ UV or $\overline{U V}-I R$, asymptotics of spinor tails.

Vertex $\overline{\mathrm{IR}}-\mathrm{IR}$ of the first type

$$
\begin{aligned}
\mathcal{M}_{\Delta_{\psi_{1}}, \Delta_{\psi_{2}}, \Delta_{\phi}}^{3 \mathrm{pt}(\mathrm{I}) \overline{\mathrm{R}}-\mathrm{IR}}\left(\vec{x}_{1}, \vec{x}_{2}, \vec{x}_{3}\right)= & \left\langle\bar{O}_{\Delta_{\psi_{1}}}^{\mathrm{IR}}\left(\vec{x}_{1}\right) O_{\Delta_{\psi_{2}}}^{\mathrm{IR}}\left(\vec{x}_{2}\right) O_{\Delta_{\phi}}\left(\vec{x}_{3}\right)\right\rangle \\
= & \int_{\mathrm{AdS}} d Z \bar{\Sigma}_{\Delta_{\psi_{1}}}^{\mathrm{IR}}\left(Z, \vec{x}_{1}\right) \\
& \times \Sigma_{\Delta_{\psi_{2}}}^{\mathrm{IR}}\left(Z, \vec{x}_{2}\right) K_{\Delta_{\phi}}\left(Z, \vec{x}_{3}\right)
\end{aligned}
$$

is quite simple because its dependence on the bulk coordinates $\left(z_{0}, \vec{z}\right)$ drops out from the spinor numerator in (32). Really, according to (15) and (16),

$$
\begin{aligned}
& \bar{\Sigma}_{\Delta_{\psi_{1}}}^{\mathrm{IR}}\left(Z, \vec{x}_{1}\right) \Sigma_{\Delta_{\psi_{2}}}^{\mathrm{IR}}\left(Z, \vec{x}_{2}\right) \\
& \sim \frac{\Pi_{+}\left[z_{0}+\vec{\gamma}\left(\vec{z}-\vec{x}_{1}\right)\right]}{\sqrt{z_{0}}} \cdot \frac{\left[z_{0}-\vec{\gamma}\left(\vec{z}-\vec{x}_{2}\right)\right] \Pi_{-}}{\sqrt{z_{0}}}=\vec{\gamma}\left(\vec{x}_{2}-\vec{x}_{1}\right) \Pi_{-}
\end{aligned}
$$

(I remind the reader that $\Pi_{+} \Pi_{-}=0$ and $\Pi_{+} \vec{\gamma} \Pi_{-}=\vec{\gamma} \Pi_{-}$). In the same way in the $\overline{\mathrm{UV}}-\mathrm{UV}$ case,

$$
\bar{\Sigma}_{d-\Delta_{\psi_{1}}}^{\mathrm{UV}}\left(Z, \vec{x}_{1}\right) \Sigma_{d-\Delta_{\psi_{2}}}^{\mathrm{UV}}\left(Z, \vec{x}_{2}\right) \sim \vec{\gamma}\left(\vec{x}_{1}-\vec{x}_{2}\right) \Pi_{+} .
$$

After substitution in (32) of three bulk-to-boundary propagators from (15), (16), and (6) with account of (33), we obtain

$$
\begin{aligned}
& \mathcal{M}_{\Delta_{\psi_{1}}, \Delta_{\psi_{2}}, \Delta_{\phi}}^{\mathrm{jpt}(\mathrm{I}) \overline{\mathrm{R}}-\mathrm{IR}}\left(\vec{x}_{1}, \vec{x}_{2}, \vec{x}_{3}\right) \\
& =\left(\prod_{i=1}^{2} \hat{C}_{\Delta_{\psi_{i}}}\right) C_{\Delta_{\phi}} \mathrm{D}_{\Delta_{\psi_{1}}+\frac{1}{2}, \Delta_{\psi_{2}}+\frac{1}{2}, \Delta_{\phi}}^{(0)}\left(\vec{x}_{1}, \vec{x}_{2}, \vec{x}_{3}\right) \vec{\gamma}\left(\vec{x}_{2}-\vec{x}_{1}\right) \Pi_{-} \\
& =B^{(\mathrm{I})}\left(\Delta_{\psi_{1}}, \Delta_{\psi_{2}}, \Delta_{\phi} ; 1 / 2\right) \cdot \frac{\vec{\gamma}\left(\vec{x}_{2}-\vec{x}_{1}\right) \Pi_{-}}{P_{12}^{\delta_{12}^{(I)}} P_{13}^{\delta_{13}^{(I)}} P_{23}^{\delta_{23}^{(I)}}}
\end{aligned}
$$

where [see (22) for $\mathrm{D}^{(0)}$ ] 


$$
\begin{aligned}
B^{(\mathrm{I})}\left(\Delta_{\psi_{1}}, \Delta_{\psi_{2}}, \Delta_{\phi} ; 1 / 2\right)= & \frac{\hat{C}_{\Delta_{\psi_{1}}}}{\Gamma\left(\Delta_{\psi_{1}}+\frac{1}{2}\right)} \frac{\hat{C}_{\Delta_{\psi_{2}}}}{\Gamma\left(\Delta_{\psi_{2}}+\frac{1}{2}\right)} \frac{C_{\Delta_{\phi}}}{\Gamma\left(\Delta_{\phi}\right)} \\
& \cdot \frac{\pi^{d / 2}}{2} \Gamma\left(\frac{\Delta_{\psi_{1}}+\Delta_{\psi_{2}}+\Delta_{\phi}+1-d}{2}\right) \Gamma\left(\delta_{12}^{(\mathrm{I})}\right) \Gamma\left(\delta_{13}^{(\mathrm{I})}\right) \Gamma\left(\delta_{23}^{(\mathrm{I})}\right), \\
\delta_{12}^{(\mathrm{I})}= & \frac{\Delta_{\psi_{1}}+\Delta_{\psi_{2}}+1-\Delta_{\phi}}{2} ; \quad \delta_{13}^{(\mathrm{I})}=\frac{\Delta_{\psi_{1}}-\Delta_{\psi_{2}}+\Delta_{\phi}}{2} ; \quad \delta_{23}^{(\mathrm{I})}=\frac{\Delta_{\psi_{2}}-\Delta_{\psi_{1}}+\Delta_{\phi}}{2} .
\end{aligned}
$$

Formula similar to (36) is obtained for $\mathcal{M}_{d-\Delta_{\psi_{1}}, d-\Delta_{\psi_{2}}, \Delta_{\phi}}^{3 \mathrm{pt}(\mathrm{I} \overline{\mathrm{UV}}-\mathrm{UV}}\left(\vec{x}_{1}, \vec{x}_{2}, \vec{x}_{3}\right)$ with account of (34) and with replacements $\Delta_{\psi_{1,2}} \rightarrow$ $d-\Delta_{\psi_{1,2}}$ in (35), (36), and $\vec{\gamma}\left(\vec{x}_{2}-\vec{x}_{1}\right) \Pi_{-} \rightarrow \vec{\gamma}\left(\vec{x}_{1}-\vec{x}_{2}\right) \Pi_{+}$in the spinor numerator in the rhs of (35).

The simple result (35) for the IR - IR spinor-spinor-scalar vertex (of type I) was obtained in Ref. [34] in physical $\operatorname{AdS}_{d+1}$ space and in Refs. [36-38] in the formalism of embedding space.

\section{B. Spinor-spinor-scalar vertex: Type II}

Let us consider the $\overline{\mathrm{IR}}-\mathrm{UV}$ vertex

$$
\begin{aligned}
\mathcal{M}_{\Delta_{\psi_{1}}, d-\Delta_{\psi_{2}}, \Delta_{\phi}}^{3 \mathrm{pt}(\mathrm{II}) \overline{\mathrm{R}}-\mathrm{UV}}\left(\vec{x}_{1}, \vec{x}_{2}, \vec{x}_{3}\right) & =\left\langle\bar{O}_{\Delta_{\psi_{1}}}^{\mathrm{IR}}\left(\vec{x}_{1}\right) O_{d-\Delta_{\psi_{2}}}^{\mathrm{UV}}\left(\vec{x}_{2}\right) O_{\Delta_{\phi}}\left(\vec{x}_{3}\right)\right\rangle \\
& =\int_{\mathrm{AdS}} d Z \bar{\Sigma}_{\Delta_{\psi_{1}}}^{\mathrm{IR}}\left(Z, \vec{x}_{1}\right) \Sigma_{d-\Delta_{\psi_{2}}}^{\mathrm{UV}}\left(Z, \vec{x}_{2}\right) K_{\Delta_{\phi}}\left(Z, \vec{x}_{3}\right) .
\end{aligned}
$$

In this case, the spinor numerator in (37) differs from the one in (33) and essentially depends on $z_{0}, \vec{z}$ :

$$
\begin{aligned}
\bar{\Sigma}_{\Delta_{\psi_{1}}}^{\mathrm{IR}}\left(Z, \vec{x}_{1}\right) \Sigma_{d-\Delta_{\psi_{2}}}^{\mathrm{UV}}\left(Z, \vec{x}_{2}\right) & \sim \frac{\Pi_{+}\left[z_{0}+\vec{\gamma}\left(\vec{z}-\vec{x}_{1}\right)\right]}{\sqrt{z_{0}}} \cdot \frac{\left[z_{0}+\vec{\gamma}\left(\vec{z}-\vec{x}_{2}\right)\right] \Pi_{+}}{\sqrt{z}_{0}} \\
& =\left\{\frac{1}{2}\left[-\frac{P_{12}}{z_{0}}+Q^{-1}\left(Z, \vec{x}_{1}\right)+Q^{-1}\left(Z, \vec{x}_{2}\right)\right]+\frac{\left(\vec{z}-\vec{x}_{1}\right)^{\alpha}\left(\vec{z}-\vec{x}_{2}\right)^{\beta} S^{\alpha \beta}}{z_{0}}\right\} \Pi_{+}
\end{aligned}
$$

where $P_{12} \equiv P_{x_{1} x_{2}}, Q(Z, \vec{x})$ see in (6) and $S^{\alpha \beta}$ in (31).

The same expression, with the only change of $\Pi_{+} \rightarrow \Pi_{-}$in the rhs of (38), is valid for the spinor numerator of $\bar{\Sigma}_{d-\Delta_{\psi_{1}}}^{\mathrm{UV}}\left(Z, \vec{x}_{1}\right) \Sigma_{\Delta_{\psi_{2}}}^{\mathrm{IR}}\left(Z, \vec{x}_{2}\right)$.

Substitution in (37) of the bulk-to-boundary propagators from (16), (17), and (6) with account of (38) gives for the spinorspinor-scalar vertex of type II

$$
\begin{aligned}
\mathcal{M}_{\Delta_{\psi_{1}}, d-\Delta_{\psi_{2}}, \Delta_{\phi}}^{3 \mathrm{pt}(\mathrm{II}) \overline{\mathrm{R}}-\mathrm{UV}}\left(\vec{x}_{1}, \vec{x}_{2}, \vec{x}_{3}\right)= & \frac{1}{2} \hat{C}_{\Delta_{\psi_{1}}} \hat{C}_{d-\Delta_{\psi_{2}}} C_{\Delta_{\phi}}\left[-P_{12} \mathrm{D}_{\Delta_{\psi_{1}}+\frac{1}{2}, d-\Delta_{\psi_{2}}+\frac{1}{2}, \Delta_{\phi}}^{(1)}\left(\vec{x}_{1}, \vec{x}_{2}, \vec{x}_{3}\right)\right. \\
& +\mathrm{D}_{\Delta_{\psi_{1}}-\frac{1}{2}, d-\Delta_{\psi_{2}}+\frac{1}{2}, \Delta_{\phi}}^{(0)}\left(\vec{x}_{1}, \vec{x}_{2}, \vec{x}_{3}\right)+\mathrm{D}_{\Delta_{\psi_{1}}+\frac{1}{2}, d-\Delta_{\psi_{2}}-\frac{1}{2}, \Delta_{\phi}}^{(0)}\left(\vec{x}_{1}, \vec{x}_{2}, \vec{x}_{3}\right) \\
& \left.+\frac{\pi^{d / 2}}{2} \frac{\Gamma\left(\frac{\Delta_{\psi_{1}}-\Delta_{\psi_{2}}+\Delta_{\phi}}{2}\right) \Gamma\left(\delta_{12}^{(\mathrm{II})}\right) \Gamma\left(\delta_{13}^{\mathrm{II})}\right) \Gamma\left(\delta_{23}^{(\mathrm{II})}\right)}{\Gamma\left(\Delta_{\psi_{1}}+\frac{1}{2}\right) \Gamma\left(d-\Delta_{\psi_{2}}+\frac{1}{2}\right) \Gamma\left(\Delta_{\phi}\right)} \cdot \vec{x}_{13}^{\alpha} \vec{x}_{23}^{\beta} S^{\alpha \beta}\right] \Pi_{+},
\end{aligned}
$$

$\vec{x}_{13}^{\alpha}=\left(\vec{x}_{1}-\vec{x}_{3}\right)^{\alpha}, \vec{x}_{23}^{\beta}=\left(\vec{x}_{2}-\vec{x}_{3}\right)^{\beta} ; \delta_{i j}^{(\mathrm{II})}$ are defined in (42) below; derivation of the $S^{\alpha \beta}$ term in the last line of (39) is similar to derivation of (24) and (29).

Then, with account of expressions (22) and (24) for $\mathrm{D}^{(0)}$ and $\mathrm{D}^{(1)}$ and their relations (25), a final rather simple formula for $\mathcal{M}^{3 \mathrm{pt}(\mathrm{II}) \overline{\mathrm{R}}-\mathrm{UV}}$ is obtained,

$$
\begin{aligned}
\mathcal{M}_{\Delta_{\psi_{1}}, d-\Delta_{\psi_{2}}, \Delta_{\phi}}^{3 \mathrm{pt}(\mathrm{II}) \overline{\mathrm{R}}-\mathrm{UV}}\left(\vec{x}_{1}, \vec{x}_{2}, \vec{x}_{3}\right)= & B^{(\mathrm{II})}\left(\Delta_{\psi_{1}}, d-\Delta_{\psi_{2}}, \Delta_{\phi} ; 1 / 2\right) \\
& \cdot \frac{-P_{12}+P_{13}+P_{23}+\left(\vec{x}_{1}-\vec{x}_{3}\right)^{\alpha}\left(\vec{x}_{2}-\vec{x}_{3}\right)^{\beta} S^{\alpha \beta}}{P_{12}^{\delta_{12}^{(\mathrm{II})}} P_{13}^{\delta_{13}^{(I I)}} P_{23}^{\delta_{23}^{(\mathrm{II}}}} \Pi_{+},
\end{aligned}
$$


where

$$
\begin{aligned}
B^{(\mathrm{II})}\left(\Delta_{\psi_{1}}, d-\Delta_{\psi_{2}}, \Delta_{\phi} ; 1 / 2\right)= & \frac{\hat{C}_{\Delta_{\psi_{1}}}}{\Gamma\left(\Delta_{\psi_{1}}+\frac{1}{2}\right)} \frac{\hat{C}_{d-\Delta_{\psi_{2}}}}{\Gamma\left(d-\Delta_{\psi_{2}}+\frac{1}{2}\right)} \frac{C_{\Delta_{\phi}}}{\Gamma\left(\Delta_{\phi}\right)} \\
& \cdot \frac{\pi^{d / 2}}{4} \Gamma\left(\frac{\Delta_{\psi_{1}}-\Delta_{\psi_{2}}+\Delta_{\phi}}{2}\right) \Gamma\left(\delta_{12}^{(\mathrm{II})}\right) \Gamma\left(\delta_{13}^{(\mathrm{II})}\right) \Gamma\left(\delta_{23}^{(\mathrm{II})}\right),
\end{aligned}
$$

and

$$
\delta_{12}^{(\mathrm{II})}=\frac{\Delta_{\psi_{1}}+d-\Delta_{\psi_{2}}-\Delta_{\phi}}{2} ; \quad \delta_{13}^{(\mathrm{II})}=\frac{\Delta_{\psi_{1}}+\Delta_{\phi}-\left(d-\Delta_{\psi_{2}}\right)+1}{2} ; \quad \delta_{23}^{(\mathrm{II})}=\frac{\left(d-\Delta_{\psi_{2}}\right)+\Delta_{\phi}-\Delta_{\psi_{1}}+1}{2} .
$$

Expression for $\mathcal{M}_{d-\Delta_{\psi_{1}}, \Delta_{\psi_{2}}, \Delta_{\phi}}^{3 \mathrm{pt}(\mathrm{II} \overline{\mathrm{UV}}-\mathrm{IR}}\left(\vec{x}_{1}, \vec{x}_{2}, \vec{x}_{3}\right)$ is obtained from (40)-(42) with the simple replacements $\Delta_{\psi_{1}} \rightarrow d-\Delta_{\psi_{1}}$ and $d-\Delta_{\psi_{2}} \rightarrow \Delta_{\psi_{2}}$ together with $\Pi_{+} \rightarrow \Pi_{-}$in the rhs of (40).

\section{SPINOR HARMONIC BUBBLES}

\section{A. Scalar bubble formed by two spinors}

The Fermionic bubble diagram of the scalar field on AdS was first calculated in Ref. [39] in the formalism of embedding space. This one-loop contribution to the two-point correlator of scalar field $\phi(Z)$ is generated by its bulk coupling $g \phi(Z) \bar{\psi}(Z) \chi(Z)$ with two spinor fields; it is formed by the bulk-to-boundary propagators of scalar field $K_{\phi}(6)$ and bulk Green's functions of spinor fields $S_{\psi}, S_{\chi}(19)$ :

$$
\mathcal{M}_{\Delta_{\phi} \mid \Delta_{\psi} \Delta_{X}}^{2 \mathrm{pt}\left(0 \mid \frac{11}{22}\right)}\left(\vec{x}_{1}, \vec{x}_{2}\right)=g^{2} \iint K_{\Delta_{\phi}}\left(X ; \vec{x}_{1}\right) \operatorname{Tr}\left[S_{\Delta_{\psi}}(X, Y) S_{\Delta_{\chi}}(Y, X)\right] K_{\Delta_{\phi}}\left(Y ; \vec{x}_{2}\right)
$$

(trace $\operatorname{Tr}$ is over spinor indices, and bulk integrals over $X, Y$ are supposed).

Following the approach of Ref. [40] in which double integral spectral representations of bubbles of fields of any integer spin were considered and referring to the spectral representation (19) of the spinor Green's function, the double integral spectral representation of bubble (43) may be put down,

$$
\mathcal{M}_{\Delta_{\phi} \mid \Delta_{\psi} \Delta_{\chi}}^{2 \mathrm{pt}\left(0 \mid \frac{11}{22}\right)}\left(\vec{x}_{1}, \vec{x}_{2}\right)=-\frac{1}{4 \pi^{2}} \iint \frac{d c d \bar{c} \mathcal{H}_{\Delta_{\phi} \mid \frac{d}{2}+i c, \frac{d}{2}+i \bar{c}}^{2 p t\left(0 \frac{11}{2}\right)}\left(\vec{x}_{1}, \vec{x}_{2}\right)}{\left[c+i\left(\Delta_{\psi}-\frac{d}{2}\right)\right]\left[\bar{c}+i\left(\Delta_{\chi}-\frac{d}{2}\right)\right]},
$$

where, taking into account the proportionality of spinor harmonic function $\Omega_{c, \frac{1}{2}}$ entering spectral representation (19) to the difference of Green's functions $\tilde{S}(20)$, we introduced in the numerator of the integrand in (44) the "harmonic bubble" $\mathcal{H}$ that is built by the replacement in (43) of two bulk spinor Green's functions with the corresponding differences $\tilde{S}$ (20):

$$
\mathcal{H}_{\Delta_{\phi} \mid \Delta_{\psi} \Delta_{X}}^{2 \mathrm{pt}\left(0 \mid \frac{11}{22}\right)}\left(\vec{x}_{1}, \vec{x}_{2}\right)=g^{2} \iint K_{\Delta_{\phi}}\left(X, \vec{x}_{1}\right) \operatorname{Tr}\left[\tilde{S}_{\Delta_{\psi}}(X, Y) \tilde{S}_{\Delta_{X}}(Y, X)\right] K_{\Delta_{\phi}}\left(Y, \vec{x}_{2}\right) .
$$

Surely, to use this expression in spectral representation (44), the replacements $\Delta_{\psi} \rightarrow d / 2+i c, \Delta_{\chi} \rightarrow d / 2+i \bar{c}$ must be performed in it.

Substitution in (45) of split representations (20) of $\tilde{S}_{\Delta_{\psi}}$ and $\tilde{S}_{\Delta_{\gamma}}$ gives two spinor-spinor-scalar vertices of type I (35) with their convolution over two boundary points $\vec{x}_{a}$ and $\vec{x}_{b}$. Thus, the rhs of (45) takes the form

$$
\begin{aligned}
& g^{2} \operatorname{Tr} \iint d \vec{x}_{a} d \vec{x}_{b}\left[\int K_{\Delta_{\phi}}\left(X, \vec{x}_{1}\right) \bar{\Sigma}_{\Delta_{\chi}}^{\mathrm{IR}}\left(X, \vec{x}_{b}\right) \Sigma_{\Delta_{\psi}}^{\mathrm{IR}}\left(X, \vec{x}_{a}\right) d X\right] \cdot\left[\int \bar{\Sigma}_{d-\Delta_{\psi}}^{\mathrm{UV}}\left(Y, \vec{x}_{a}\right) \Sigma_{d-\Delta_{\chi}}^{\mathrm{UV}}\left(Y, \vec{x}_{b}\right) K_{\Delta_{\phi}}\left(Y, \vec{x}_{2}\right) d Y\right] \\
& \quad=g^{2} \operatorname{Tr} \iint d \vec{x}_{a} d \vec{x}_{b} \mathcal{M}_{\Delta_{\chi}, \Delta_{\psi}, \Delta_{\phi}}^{3 \mathrm{pt}(\mathrm{I}) \overline{\mathrm{R}}-\mathrm{IR}}\left(\vec{x}_{b}, \vec{x}_{a}, \vec{x}_{1}\right) \mathcal{M}_{d-\Delta_{\psi}, d-\Delta_{\chi}, \Delta_{\phi}}^{3 \mathrm{pt}(\mathrm{I}) \overline{\mathrm{UV}}\left(\vec{x}_{a}, \vec{x}_{b}, \vec{x}_{2}\right) .}
\end{aligned}
$$

Using (35) for $\mathcal{M}^{3 \mathrm{pt}(\mathrm{I}) \overline{\mathrm{IR}}-\mathrm{IR}}$ and a similar expression for $\mathcal{M}^{\mathrm{Bpt}(\mathrm{I}) \overline{\mathrm{UV}}-\mathrm{UV}}$ with evident changes of arguments, taking into account 
$\operatorname{Tr}\left[\vec{\gamma}\left(\vec{x}_{a}-\vec{x}_{b}\right) \Pi_{-} \vec{\gamma}\left(\vec{x}_{a}-\vec{x}_{b}\right) \Pi_{+}\right]=P_{a b} \operatorname{Tr} \Pi_{+}=\frac{P_{a b}}{2} \cdot \operatorname{dim} \gamma$

[ $\operatorname{dim} \gamma=\operatorname{Tr}[\hat{1}]$ is equal to $d$ for $d$ even and equal to $(d-1)$ for $d$ odd], and performing standard conformal integral (26) over $\vec{x}_{b}$ the following expression for vertex (45) is obtained:

$$
\begin{aligned}
& \mathcal{H}_{\Delta_{\phi} \mid \Delta_{\psi}}^{2 \mathrm{pt}\left(0 \mid \frac{11}{22}\right)}\left(\vec{x}_{1}, \vec{x}_{2}\right) \\
&=\frac{g^{2} \operatorname{dim} \gamma}{2} \frac{1}{P_{12}^{\Delta_{\phi}-\frac{d}{2}}} \int \frac{d \vec{x}_{a}}{P_{1 a}^{\frac{d}{2}} P_{2 a}^{\frac{d}{2}}} \\
& \cdot B^{(\mathrm{I})}\left(\Delta_{\psi}, \Delta_{\chi}, \Delta_{\phi} ; 1 / 2\right) B^{(\mathrm{I})}\left(d-\Delta_{\psi}, d-\Delta_{\chi}, \Delta_{\phi} ; 1 / 2\right) \\
& \cdot A\left(\frac{\Delta_{\psi}-\Delta_{\chi}+\Delta_{\phi}}{2}, \frac{\Delta_{\chi}-\Delta_{\psi}+\Delta_{\phi}}{2}, d-\Delta_{\phi}\right)
\end{aligned}
$$

$A, B^{(\mathrm{I})}$ see in (28), (36).

Typical for conformal theories, the divergent integral in (47) was analyzed in Ref. [40]; here, the dimensional regularization is chosen when in general formulas (27) and (28) one takes

$$
d \rightarrow d^{*}=d+\epsilon, \quad \beta_{1}=\beta_{2}=\frac{d}{2} .
$$

We leave in integral (47) only most divergent term $\sim \epsilon^{-1}$ and absorb it in the "bare" coupling constant $g$, defining in this way the renormalized coupling as:

$$
g_{R}^{2}=g^{2} P_{12}^{\frac{d}{2}} \int \frac{d \vec{x}_{a}}{P_{1 a}^{\frac{d}{2}} P_{2 a}^{\frac{d}{2}}}=g^{2} \frac{4 \pi^{\frac{d}{2}}}{\Gamma\left(\frac{d}{2}\right)} \frac{1}{\epsilon} .
$$

Thus, using (48) and deciphering $A(28), B^{(\mathrm{I})}$ (36), and $\hat{C}_{\Delta_{\psi \cdot \varkappa}}$ (13) that enter the expression for $B^{(\mathrm{I})}$, we finally get

$$
\mathcal{H}_{\Delta_{\phi} \mid \Delta_{\psi} \Delta_{\chi}}^{2 \mathrm{pt}\left(0 \mid \frac{11}{22}\right)}\left(\vec{x}_{1}, \vec{x}_{2}\right)=\frac{C_{\Delta_{\phi}}}{P_{12}^{\Delta_{\phi}}} g_{R}^{2} \frac{\operatorname{dim} \gamma}{16 \pi^{d}} \frac{\mathbf{R}\left(\Delta_{\psi}, \Delta_{\chi} ; \Delta_{\phi}\right)}{F^{(0)}\left(\Delta_{\phi}\right)},
$$

where

$$
F^{(0)}\left(\Delta_{\phi}\right)=\frac{\Gamma\left(\Delta_{\phi}\right) \Gamma\left(d-\Delta_{\phi}\right)}{\Gamma\left(\Delta_{\phi}-\frac{d}{2}\right) \Gamma\left(\frac{d}{2}-\Delta_{\phi}\right)},
$$

and

$$
\begin{aligned}
\mathbf{R}\left(\Delta_{\psi}, \Delta_{\chi} ; \Delta_{\phi}\right) \\
=\frac{\Gamma\left(\frac{\Delta_{\psi}+\Delta_{\chi}+\Delta_{\phi}-d+1}{2}\right) \Gamma\left(\frac{2 d-\Delta_{\psi}-\Delta_{\chi}-\Delta_{\phi}+1}{2}\right)}{\Gamma\left(\frac{1}{2}+\Delta_{\psi}-\frac{d}{2}\right) \Gamma\left(\frac{1}{2}+\frac{d}{2}-\Delta_{\psi}\right)} \\
\quad \cdot \frac{\Gamma\left(\frac{\Delta_{\psi}-\Delta_{\chi}+\Delta_{\phi}}{2}\right) \Gamma\left(\frac{\Delta_{\chi}-\Delta_{\psi}+\Delta_{\phi}}{2}\right) \Gamma\left(\frac{\Delta_{\psi}+\Delta_{\chi}-\Delta_{\phi}+1}{2}\right)}{\Gamma\left(\frac{1}{2}+\Delta_{\chi}-\frac{d}{2}\right) \Gamma\left(\frac{1}{2}+\frac{d}{2}-\Delta_{\chi}\right)} \\
\quad \cdot \frac{\Gamma\left(\frac{d+\Delta_{\psi}-\Delta_{\chi}-\Delta_{\phi}}{2}\right) \Gamma\left(\frac{d+\Delta_{\chi}-\Delta_{\psi}-\Delta_{\phi}}{2}\right) \Gamma\left(\frac{d-\Delta_{\psi}-\Delta_{\chi}+\Delta_{\phi}+1}{2}\right)}{\Gamma\left(\frac{d}{2}-\Delta_{\phi}\right) \Gamma\left(1+\Delta_{\phi}-\frac{d}{2}\right)},
\end{aligned}
$$

or expressing $\mathbf{R}$ through bulk masses of spinors $m_{\psi}=$ $\Delta_{\psi}-d / 2$ and $m_{\chi}=\Delta_{\chi}-d / 2$ [see (12)] and Bessel functions' order $\nu=\Delta_{\phi}-d / 2$ [see (5)] for scalar field:

$$
\begin{aligned}
\mathbf{R}\left(\Delta_{\psi}, \Delta_{\chi} ; \Delta_{\phi}\right) & \\
= & \frac{\Gamma\left(\frac{d}{4}+\alpha\right) \Gamma\left(\frac{d}{4}-\alpha\right) \Gamma\left(\frac{d}{4}+\beta\right) \Gamma\left(\frac{d}{4}-\beta\right)}{\Gamma\left(\frac{1}{2}+m_{\psi}\right) \Gamma\left(\frac{1}{2}-m_{\psi}\right)} \\
& \cdot \frac{\Gamma\left(\frac{1}{2}+\frac{d}{4}+\gamma\right) \Gamma\left(\frac{1}{2}+\frac{d}{4}-\gamma\right) \Gamma\left(\frac{1}{2}+\frac{d}{4}+\delta\right) \Gamma\left(\frac{1}{2}+\frac{d}{4}-\delta\right)}{\Gamma\left(\frac{1}{2}+m_{\chi}\right) \Gamma\left(\frac{1}{2}-m_{\chi}\right) \Gamma(-\nu) \Gamma(1+\nu)}, \\
\alpha= & \frac{m_{\psi}-m_{\chi}+\nu}{2}, \quad \beta=\frac{m_{\chi}-m_{\psi}+\nu}{2}, \quad \delta=\frac{m_{\psi}+m_{\chi}-\nu}{2} .
\end{aligned}
$$

$\mathbf{R}$ is the main function in our analysis. It can be seen that for even $d$ it is expressed in terms of elementary trigonometric or hyperbolic [for imaginary arguments, like in spectral representations (8), (19)] functions. For example, for $d=2$,

$$
\mathbf{R}\left(\Delta_{\psi}, \Delta_{\chi} ; \Delta_{\phi}\right)=-\frac{\pi \gamma \delta \cos \pi m_{\psi} \cos \pi m_{\chi} \sin \pi \nu}{\cos \pi \alpha \cos \pi \beta \sin \pi \gamma \sin \pi \delta},
$$

and for $d=4$,

$$
\begin{aligned}
& \mathbf{R}\left(\Delta_{\psi}, \Delta_{\chi} ; \Delta_{\phi}\right) \\
& =-\frac{\pi \alpha \beta\left(\frac{1}{4}-\gamma^{2}\right)\left(\frac{1}{4}-\delta^{2}\right) \cos \pi m_{\psi} \cos \pi m_{\chi} \sin \pi \nu}{\sin \pi \alpha \sin \pi \beta \cos \pi \gamma \cos \pi \delta} .
\end{aligned}
$$

As could be expected, dependence on coordinates of the harmonic bubble (49), and hence of the full bubble (44), is the same as that of the elementary scalar conformal correlator (7); it is singled out in front of the rhs of (49).

\section{B. Spinor bubble formed by spinor and scalar}

Generated by the same bulk Yukawa coupling like in the previous subsection, the one-loop two-point contribution to the conformal correlator of spinor field $\psi(Z)$ when the loop is formed by spinor field $\chi(Z)$ and scalar field $\phi(Z)$ has the form

$$
\begin{aligned}
\mathcal{M}_{\Delta_{\psi} \mid \Delta_{\chi}, \Delta_{\phi}}^{2 \mathrm{pt}\left(\frac{1}{2} \frac{1}{2} 0\right)}\left(\vec{x}_{1}, \vec{x}_{2}\right)= & g^{2} \iint \bar{\Sigma}_{\Delta_{\Delta^{\prime}}}^{\mathrm{IR}}\left(X, \vec{x}_{1}\right) S_{\Delta_{\chi}}(X, Y) G_{\Delta_{\phi}}(X, Y) \\
& \times \Sigma_{\Delta_{\psi}}^{\mathrm{IR}}\left(Y, \vec{x}_{2}\right),
\end{aligned}
$$

where spinor bulk-to-boundary propagators $\Sigma_{\Delta_{\psi}}^{\mathrm{IR}}$ and $\bar{\Sigma}_{\Delta_{\psi}}^{\mathrm{IR}}$ are given in (15) and (16) and Green's functions $G_{\Delta_{\phi}}$ and $S_{\Delta_{\chi}}$ are given in (8) and (19) (the IR option is meant, that is, $\Delta_{\phi}>d / 2$ and $\Delta_{\chi}>d / 2$; see (5) and (12)].

Following the logic of the previous section, with account of spectral representations of scalar (8) and spinor (19) 
Green's functions and the proportionality of corresponding harmonic functions to differences $\tilde{G}(10)$ and $\tilde{S}$ (20) of IR and UV scalar and spinor Green's functions, the double-integral spectral representation of the one-loop spinor correlator (53) is obtained,

$$
\mathcal{M}_{\Delta_{\psi} \mid \Delta_{\chi} \Delta_{\phi}}^{2 \mathrm{pt}\left(\frac{1}{2} \frac{1}{2} 0\right)}\left(\vec{x}_{1}, \vec{x}_{2}\right)=-\frac{1}{4 \pi^{2}} \iint \frac{d c \bar{c} d \bar{c} \mathcal{H}_{\Delta_{\psi} \mid \frac{d}{2}+i c, \frac{d}{2}+i \bar{c}}^{2 \mathrm{pt}\left(\frac{1}{2} \frac{1}{2} 0\right)}}{\left[c+i\left(\Delta_{\chi}-\frac{d}{2}\right)\right]\left[\bar{c}^{2}+\left(\Delta_{\phi}-\frac{d}{2}\right)^{2}\right]},
$$

where harmonic bubble $\mathcal{H}^{2 \mathrm{pt}\left(\frac{1}{2} \frac{1}{2} 0\right)}$ in the numerator is again built by the replacement in (53) of two bulk Green's functions $G$ and $S$ with corresponding differences $\tilde{G}(10)$ and $\tilde{S}(20)$ :

$$
\mathcal{H}_{\Delta_{\psi} \mid \Delta_{\chi} \Delta_{\phi}}^{2 \mathrm{pt}\left(\frac{1}{2} \frac{1}{2} 0\right)}\left(\vec{x}_{1}, \vec{x}_{2}\right)=g^{2} \iint \bar{\Sigma}_{\Delta_{\psi}}^{\mathrm{IR}}\left(X, \vec{x}_{1}\right) \tilde{S}_{\Delta_{\chi}}(X, Y) \tilde{G}_{\Delta_{\phi}}(X, Y) \Sigma_{\Delta_{\psi}}^{\mathrm{IR}}\left(Y, \vec{x}_{2}\right) .
$$

To use it in spectral representation (54), the replacements $\Delta_{\chi} \rightarrow d / 2+i c$ and $\Delta_{\phi} \rightarrow d / 2+i \bar{c}$ must be performed in (55).

Substitution here of split representations of $\tilde{G}(10)$ and $\tilde{S}$ (20) gives for the rhs of (55)

$$
\begin{gathered}
g^{2}\left(d-2 \Delta_{\phi}\right) \iint d \vec{x}_{a} d \vec{x}_{b}\left[\int \bar{\Sigma}_{\Delta_{\psi}}^{\mathrm{IR}}\left(X, \vec{x}_{1}\right) \Sigma_{d-\Delta_{\chi}}^{\mathrm{UV}}\left(X, \vec{x}_{a}\right) K_{\Delta_{\phi}}\left(X, \vec{x}_{b}\right) d X\right] \\
\cdot\left[\int \bar{\Sigma}_{\Delta_{\chi}}^{\mathrm{IR}}\left(Y, \vec{x}_{a}\right) \Sigma_{\Delta_{\psi}}^{\mathrm{IR}}\left(Y, \vec{x}_{2}\right) K_{d-\Delta_{\phi}}\left(Y, \vec{x}_{b}\right) d Y\right]=g^{2}\left(d-2 \Delta_{\phi}\right) \\
\cdot \int d \vec{x}_{a} \int d \vec{x}_{b} \mathcal{M}_{\Delta_{\psi}, d-\Delta_{\chi}, \Delta_{\phi}}^{3 \mathrm{pt}(\mathrm{II}) \overline{\mathrm{R}}-\mathrm{UV}}\left(\vec{x}_{1}, \vec{x}_{a}, \vec{x}_{b}\right) \mathcal{M}_{\Delta_{\chi}, \Delta_{\psi}, d-\Delta_{\phi}}^{3 \mathrm{pt}(\mathrm{I}) \overline{\mathrm{R}}-\mathrm{IR}}\left(\vec{x}_{a}, \vec{x}_{2}, \vec{x}_{b}\right) ;
\end{gathered}
$$

here, the last equality follows from expressions (32) and (37) for $\mathcal{M}^{3 \mathrm{pt}(\mathrm{I})}$ and $\mathcal{M}^{3 \mathrm{pt}(\mathrm{II})}$. After the evident changes of variables and arguments in final formulas (35)-(36) and (40)-(42) for these vertices, Eq. (56) comes to

$$
\begin{aligned}
\mathcal{H}_{\Delta_{\psi} \mid \Delta_{\chi} \Delta_{\phi}}^{2 \mathrm{pt}\left(\frac{1}{2} \frac{1}{2} 0\right)}\left(\vec{x}_{1}, \vec{x}_{2}\right)= & B^{(\mathrm{II})}\left(\Delta_{\psi}, d-\Delta_{\chi}, \Delta_{\phi} ; 1 / 2\right) B^{(\mathrm{I})}\left(\Delta_{\chi}, \Delta_{\psi}, d-\Delta_{\phi} ; 1 / 2\right) \\
& \cdot g^{2}\left(d-2 \Delta_{\phi}\right) \int d \vec{x}_{a} I_{b}\left(\vec{x}_{1}, \vec{x}_{2}, \vec{x}_{a}\right) \frac{\Pi_{+} \vec{\gamma}\left(\vec{x}_{2}-\vec{x}_{a}\right) \Pi_{-}}{P_{1 a}^{\delta_{1 a}^{(\mathrm{II})}} P_{2 a}^{\delta_{2 a}^{(I)}}},
\end{aligned}
$$

where $B^{(\mathrm{I})}$ and $B^{(\mathrm{II})}$ (see (36) and (41)] and $I_{b}\left(\vec{x}_{1}, \vec{x}_{2}, \vec{x}_{a}\right)$ is an integral over $\vec{x}_{b}$,

$$
\begin{aligned}
& I_{b}\left(\vec{x}_{1}, \vec{x}_{2}, \vec{x}_{a}\right)=\int d \vec{x}_{b} \frac{\left[-P_{1 a}+P_{1 b}+P_{a b}+\left(\vec{x}_{1}-\vec{x}_{b}\right)^{\alpha}\left(\vec{x}_{a}-\vec{x}_{b}\right)^{\beta} S^{\alpha \beta}\right]}{P_{1 b}^{\delta_{1 b}^{(I)}} P_{2 b}^{\delta_{2 b}^{(I)}} P_{a b}^{\left(\delta_{a b}^{(I)}+\delta_{a b}^{(I)}\right)}}
\end{aligned}
$$

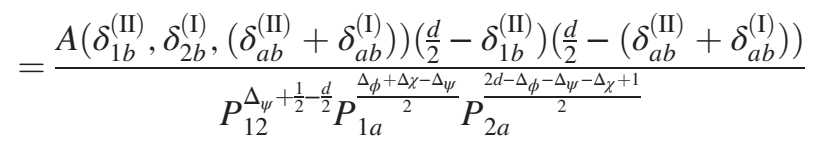

$$
\begin{aligned}
& \cdot\left[-P_{1 a}+P_{12}+P_{2 a}-\left(\vec{x}_{1}-\vec{x}_{a}\right)^{\alpha}\left(\vec{x}_{2}-\vec{x}_{a}\right)^{\beta} S^{\alpha \beta}\right],
\end{aligned}
$$

where $A$ and $S^{\alpha \beta}$ are given in (28) and (31). The values of six exponents $\delta_{i j}$ in (57)-(58) corresponding to exponents in (36) for the vertex of type I and to exponents in (42) for the vertex of type II are as follows:

$$
\delta_{2 a}^{(\mathrm{I})}=\frac{\Delta_{\psi}+\Delta_{\chi}+\Delta_{\phi}+1-d}{2}, \quad \delta_{2 b}^{(\mathrm{I})}=\frac{\Delta_{\psi}-\Delta_{\chi}-\Delta_{\phi}+d}{2}, \quad \delta_{a b}^{(\mathrm{I})}=\frac{\Delta_{\chi}-\Delta_{\psi}+d-\Delta_{\phi}}{2},
$$

and

$$
\delta_{1 a}^{(\mathrm{II})}=\frac{\Delta_{\psi}+d-\Delta_{\chi}-\Delta_{\phi}}{2}, \quad \delta_{1 b}^{(\mathrm{II})}=\frac{\Delta_{\psi}+\Delta_{\phi}-\left(d-\Delta_{\chi}\right)+1}{2}, \quad \delta_{a b}^{(\mathrm{II})}=\frac{\left(d-\Delta_{\chi}\right)+\Delta_{\phi}-\Delta_{\psi}+1}{2} .
$$


It is seen from (59) and (60) that

$$
\delta_{1 b}^{(\mathrm{II})}+\delta_{2 b}^{(\mathrm{I})}+\delta_{a b}^{(\mathrm{II})}+\delta_{a b}^{(\mathrm{I})}=d+1 .
$$

In the calculation of $I_{b}$ (58), it was taken into account that conformal integrals over $\vec{x}_{b}$ corresponding to the second $\left(P_{1 b}\right)$ and the third $\left(P_{a b}\right)$ terms in the integrand of (58) are the ordinary ones of type (26), whereas the sum of exponents of $P$ in the first $\left(P_{1 a}\right)$ term in the integrand in (58) is, according to (61), equal to $(d+1)$; this integral is less trivial [see (29)]. The integral over $\vec{x}_{b}$ of the last term on the rhs of (58) also comes to the standard conformal integral (26) with account of the asymmetry of $S^{\alpha \beta}$ and elementary identity

$$
\frac{\left(\vec{x}_{1}-\vec{x}_{b}\right)^{\alpha}\left(\vec{x}_{a}-\vec{x}_{b}\right)^{\beta} S^{\alpha \beta}}{P_{a b}^{\left(\delta_{a b}^{(I)}+\delta_{a b}^{(\mathrm{I})}\right)}}=-\frac{\left(\vec{x}_{1}-\vec{x}_{a}\right)^{\alpha} S^{\alpha \beta}}{2\left(\delta_{a b}^{(\mathrm{II})}+\delta_{a b}^{(\mathrm{I})}-1\right)} \frac{\partial}{\partial x_{a}^{\beta}}\left[\frac{1}{P_{a b}^{\delta_{a b}^{(\mathrm{II})}+\delta_{a b}^{(\mathrm{I})}-1}}\right] .
$$

Substitution of $I_{b}(58)$ in (57) with account of (59)-(61) and (28) gives

$$
\begin{aligned}
\mathcal{H}_{\Delta_{\psi} \mid}^{2 \mathrm{pt}\left(\frac{1}{2} \frac{1}{2} \frac{1}{\Delta_{\chi} \Delta_{\phi}}\right)}\left(\vec{x}_{1}, \vec{x}_{2}\right)= & g^{2}\left(d-2 \Delta_{\phi}\right) B^{(\mathrm{I})}\left(\Delta_{\psi}, \Delta_{\chi}, d-\Delta_{\phi}\right) B^{(\mathrm{II})}\left(\Delta_{\psi}, d-\Delta_{\chi}, \Delta_{\phi}\right) \frac{\tilde{A}\left(\Delta_{\psi}, \Delta_{\chi}, \Delta_{\phi}\right)}{P_{12}^{\Delta_{\psi}+\frac{1}{2}-\frac{d}{2}}} \\
& \cdot \int d \vec{x}_{a} \frac{\left[P_{2 a}+P_{12}-P_{1 a}-\left(\vec{x}_{1}-\vec{x}_{a}\right)^{\alpha}\left(\vec{x}_{2}-\vec{x}_{a}\right)^{\beta} S^{\alpha \beta}\right] \vec{\gamma}\left(\vec{x}_{2}-\vec{x}_{a}\right) \Pi_{-}}{P_{1 a}^{\frac{d}{2}} P_{2 a}^{\frac{d}{2}+1}}
\end{aligned}
$$

where

$$
\tilde{A}\left(\Delta_{\psi}, \Delta_{\chi}, \Delta_{\phi}\right)=\frac{\pi^{\frac{d}{2}} \Gamma\left(\frac{2 d-\Delta_{\psi}-\Delta_{\chi}-\Delta_{\phi}+1}{2}\right) \Gamma\left(\frac{\Delta_{\chi}-\Delta_{\psi}+\Delta_{\phi}}{2}\right) \Gamma\left(\Delta_{\psi}+\frac{1}{2}-\frac{d}{2}\right)}{\Gamma\left(\frac{\Delta_{\psi}+\Delta_{\chi}+\Delta_{\phi}-d+1}{2}\right) \Gamma\left(\frac{\Delta_{\psi}-\Delta_{\chi}+d-\Delta_{\phi}}{2}\right) \Gamma\left(d-\Delta_{\psi}+\frac{1}{2}\right)} .
$$

To take divergent conformal integrals over $\vec{x}_{a}$ in (62), the general formula (30) is applied, where according to dimensional regularization we change on the rhs $d \rightarrow d^{*}=d+\epsilon$ and put $\beta_{1}$ and $\beta_{2}$ equal to corresponding values in every integral in (62) (cf. in Ref. [40]). This gives for the last line in $(62)$

$$
\int d \vec{x}_{a}\{\ldots\}=\frac{\vec{\gamma}\left(\vec{x}_{1}-\vec{x}_{2}\right) \Pi_{-}}{P_{12}^{\frac{d}{2}}} \frac{2 \pi^{d / 2}}{\Gamma\left(\frac{d}{2}\right)} \frac{1}{\epsilon}\left(1+\frac{3}{d}\right) .
$$

The final expression for the spinor one-loop harmonic bubble (55) is obtained from (62)-(64) and expressions (36) and (41) for coefficients $B^{(\mathrm{I})}$ and $B^{(\mathrm{II})}$ with the introduction of the renormalized coupling constant (47) and with account that in general formulas (36) and (41) for coefficients $B^{(\mathrm{I})}$ and $B^{(\mathrm{II})}$ the corresponding replacements of $\delta_{i j}$ must be performed. Namely, $\delta_{12}^{(\mathrm{I})}, \delta_{13}^{(\mathrm{I})}, \delta_{23}^{(\mathrm{I})}$ [in (36)] must be changed to $\delta_{2 a}^{(\mathrm{I})}, \delta_{a b}^{(\mathrm{I})}$, and $\delta_{2 b}^{(\mathrm{I})}$ given in (59), and in the same way, $\delta_{12}^{(\mathrm{II})}$, $\delta_{13}^{(\mathrm{II})}$, and $\delta_{23}^{(\mathrm{II})}(42)$ must be changed to $\delta_{1 a}^{(\mathrm{II})}, \delta_{1 b}^{(\mathrm{II})}$, and $\delta_{a b}^{(\mathrm{II})}$ (60). The result proves to be quite simple,

$$
\begin{aligned}
\mathcal{H}_{\Delta_{\psi} \mid \Delta_{\chi} \Delta_{\phi}}^{2 \mathrm{pt}\left(\frac{1}{2} \frac{1}{2} 0\right)}\left(\vec{x}_{1}, \vec{x}_{2}\right)= & \hat{C}_{\Delta_{\psi}} \frac{\vec{\gamma}\left(\vec{x}_{1}-\vec{x}_{2}\right) \Pi_{-}}{P_{12}^{\Delta_{\psi}+\frac{1}{2}}} \\
& \times g_{R}^{2} \frac{\left(1+\frac{3}{d}\right) \mathbf{R}\left(\Delta_{\psi}, \Delta_{\chi} ; \Delta_{\phi}\right)}{32 \pi^{d}} \frac{F^{\left(\frac{1}{2}\right)}\left(\Delta_{\psi}\right)}{}
\end{aligned}
$$

where

$$
F^{\left(\frac{1}{2}\right)}\left(\Delta_{\psi}\right)=\frac{\Gamma\left(\Delta_{\psi}+\frac{1}{2}\right) \Gamma\left(d-\Delta_{\psi}+\frac{1}{2}\right)}{\Gamma\left(\Delta_{\psi}+\frac{1}{2}-\frac{d}{2}\right) \Gamma\left(\frac{d}{2}-\Delta_{\psi}+\frac{1}{2}\right)}
$$

and coefficient $\mathbf{R}\left(\Delta_{\psi}, \Delta_{\chi} ; \Delta_{\phi}\right)$ given in (51) or (52) is common for both harmonic bubbles $\mathcal{H}$ (49), (65) calculated in Secs. IV. A and IV. B. Space-time and spinor dependence in front of the rhs of (65) [and hence of the full bubble (54)] copies the same of the primary correlator (13).

As it was noted above, harmonic bubbles (49) and (65) may be used as numerators of the integrands in spectral representations (44) and (54) after the corresponding replacements in the universal function $\mathbf{R}$ (51) of conformal dimensions of intermediate fields by the integration variables $d / 2+i c, d / 2+i \bar{c}$. It is seen from the expression for $\mathbf{R}$ (51)-(52) that double-integrals (44), (54) are divergent in directions $c+\bar{c}=$ const and $c-\bar{c}=$ const [40].

In the next section, the natural subtraction of the divergences of bubbles (43), (53) will be performed.

\section{DOUBLE-TRACE ELIMINATION OF UV INFINITIES}

The UV divergence of the self-energy diagrams reflected in particular in infinity of spectral integrals (44), (54) is a conventional difficulty in quantum field theory. Here, to 
overcome this difficulty, I propose applying to the bubble diagrams the double-trace from the UV to IR flow approach used in Refs. [23-30] for the unambiguous UV-finite calculations of tadpoles and quantum vacuum energies of scalar and spinor bulk fields in spaces of arbitrary dimensions. For example, in $\mathrm{AdS}_{5}(d=4)$, expressions for tadpoles defined in this way are as follows [differences $\tilde{G}$ and $\tilde{S}$ of IR and UV Green's functions are given in (10) and (20), and we restore here the value $k_{\mathrm{AdS}}$ of the curvature of AdS space]: for the scalar tadpole [23-28],

$$
\tilde{G}_{\Delta}^{(d=4)}(Z, Z)=\frac{k_{\text {AdS }}^{3}}{12 \pi^{2}}(\Delta-1)(\Delta-2)(\Delta-3),
$$

and for the spinor tadpole [29,30],

$$
\tilde{S}_{\Delta_{\psi}=2+m}^{(d=4)}(Z, Z)=\frac{k_{\mathrm{AdS}}^{4}}{3 \pi^{2}}\left(m^{2}-\frac{1}{4}\right)\left(\frac{9}{4}-m^{2}\right) .
$$

Thus, the difference of two similar Witten diagrams built of the UV or IR bulk Green's functions proves to be finite and well defined for tadpoles, and I shall show that it is also finite and well defined for the bubbles.

Most generally, this modification of quantum diagrams means that, instead of the standard quantum generation functional, symbolically,

$$
Z[j ; G]=(\operatorname{Det} G)^{-1 / 2} e^{L_{\mathrm{int}}\left(\frac{\delta}{\delta j}\right)} e^{\left(\frac{1}{2} j G j\right)}
$$

[here, $G, L_{\text {int }}$, and $j$ are the free field Green's function, the interaction Lagrangian, and the field's source, respectively; in frames of the AdS/CFT correspondence in the generation functional of the $n$-point boundary conformal correlators, $j(\vec{x})$ is equipped with the corresponding bulkto-boundary propagator], the ratio

$$
\begin{aligned}
\tilde{Z}\left[j ; G^{\mathrm{UV}}, G^{\mathrm{IR}}\right] & =\frac{Z\left[j ; G^{\mathrm{UV}}\right]}{Z\left[j ; G^{\mathrm{IR}}\right]} \\
& =\frac{\left(\operatorname{Det} G^{\mathrm{UV}}\right)^{-1 / 2} e^{L_{\mathrm{int}}\left(i \frac{\delta}{\delta j}\right)} e^{\left(\frac{1}{2} j G^{\mathrm{UV}} j\right)}}{\left(\operatorname{Det} G^{\mathrm{IR}}\right)^{-1 / 2} e^{L_{\mathrm{int}}\left(i \frac{\delta}{\delta j}\right)} e^{\left(\frac{1}{2} j G^{\mathrm{IR}} j\right)}}
\end{aligned}
$$

of two quantum functionals determined by Green's functions $\left(G^{\mathrm{UV}}\right.$ and $\left.G^{\mathrm{IR}}\right)$ possessing two different asymptotics at the horizon must be considered as a quantum generation functional for Witten diagrams. General analysis of the ratios of quantum functionals of one and the same bulk dynamics and different boundary conditions is presented in Ref. [43].

This approach means in particular that self-energy correlators (43) and (53) are redefined as a difference $\tilde{\mathcal{M}}$ (also marked with a tilde) of conventional bubble diagrams built of the products of two UV and two IR Green's functions correspondingly,

$$
\begin{aligned}
& \tilde{\mathcal{M}}_{\Delta_{\phi} \mid \Delta_{\psi} \Delta_{\chi}}^{2 \mathrm{pt}\left(0 \mid \frac{11}{22}\right)}\left(\vec{x}_{1}, \vec{x}_{2}\right)=\mathcal{M}_{\Delta_{\phi} \mid \Delta_{\psi} \Delta_{\chi}}^{2 \mathrm{pt}\left(0 \mid \frac{11}{22}\right) \mathrm{UV}}\left(\vec{x}_{1}, \vec{x}_{2}\right)-\mathcal{M}_{\Delta_{\phi} \mid \Delta_{\psi} \Delta_{\chi}}^{2 \mathrm{pt}\left(0 \mid \frac{11}{22}\right) I \mathrm{R}}\left(\vec{x}_{1}, \vec{x}_{2}\right) \\
& =g^{2} \iint K_{\Delta_{\phi}}\left(X ; \vec{x}_{1}\right) \tilde{\Pi}_{\Delta_{\psi}, \Delta_{\chi}}^{2 \mathrm{pt}\left(0 \mid \frac{11}{22}\right)}(X, Y) K_{\Delta_{\phi}}\left(Y ; \vec{x}_{2}\right) d X d Y, \\
& \tilde{\mathcal{M}}_{\Delta_{\psi} \mid \Delta_{\chi}, \Delta_{\phi}}^{2 \mathrm{pt}\left(\frac{1}{2} \frac{1}{2} \frac{1}{2}\right)}\left(\vec{x}_{1}, \vec{x}_{2}\right)=\mathcal{M}_{\Delta_{\psi} \mid \Delta_{\chi}, \Delta_{\phi}}^{2 \mathrm{pt}\left(\frac{1}{2} \frac{1}{2}\right) \mathrm{UV}}\left(\vec{x}_{1}, \vec{x}_{2}\right)-\mathcal{M}_{\Delta_{\psi} \mid \Delta_{\chi}, \Delta_{\phi}}^{2 \mathrm{pt}\left(\frac{1}{2} \frac{1}{2} 0\right) \operatorname{IR}}\left(\vec{x}_{1}, \vec{x}_{2}\right) \\
& =g^{2} \iint \bar{\Sigma}_{\Delta_{\psi}}^{\mathrm{IR}}\left(X, \vec{x}_{1}\right) \tilde{\Pi}_{\Delta_{\chi}, \Delta_{\phi}}^{2 \mathrm{pt}\left(\frac{1}{2} \frac{1}{2} 0\right)}(X, Y) \Sigma_{\Delta_{\psi}}^{\mathrm{IR}}\left(Y, \vec{x}_{2}\right) d X d Y,
\end{aligned}
$$

where

$$
\begin{aligned}
\tilde{\Pi}_{\Delta_{\psi}, \Delta_{\chi}}^{2 \mathrm{pt}\left(\left[\frac{11}{22}\right)\right.}(X, Y)=\left\{\operatorname{Tr}\left[S_{\Delta_{\psi}}^{\mathrm{UV}} S_{\Delta_{\chi}}^{\mathrm{UV}}\right]-\operatorname{Tr}\left[S_{\Delta_{\psi}}^{\mathrm{IR}} S_{\Delta_{\chi}}^{\mathrm{IR}}\right]\right\}(X, Y) \\
=\left\{\operatorname{Tr}\left[\tilde{S}_{\Delta_{\psi}} \tilde{S}_{\Delta_{\chi}}\right]-\operatorname{Tr}\left[S_{\Delta_{\psi}}^{\mathrm{IR}} \tilde{S}_{\Delta_{\chi}}\right]-\operatorname{Tr}\left[\tilde{S}_{\Delta_{\psi}} S_{\Delta_{\chi}}^{\mathrm{IR}}\right]\right\}(X, Y), \\
\tilde{\Pi}_{\Delta_{\chi}, \Delta_{\phi}}^{2 \mathrm{pt}\left(\frac{1}{2} \frac{1}{2} 0\right)}(X, Y)=\left\{S_{\Delta_{\chi}}^{\mathrm{UV}} G_{\Delta_{\phi}}^{\mathrm{UV}}-S_{\Delta_{\chi}}^{\mathrm{IR}} G_{\Delta_{\phi}}^{\mathrm{IR}}\right\}(X, Y) \\
=\left\{\tilde{S}_{\Delta_{\chi}} \tilde{G}_{\Delta_{\phi}}-S_{\Delta_{\chi}}^{\mathrm{IR}} \tilde{G}_{\Delta_{\phi}}-\tilde{S}_{\Delta_{\chi}} G_{\Delta_{\phi}}^{\mathrm{IR}}\right\}(X, Y) .
\end{aligned}
$$

In the derivation of (71) and (72), identities $G^{\mathrm{UV}}=G^{\mathrm{IR}}-\tilde{G}$ and $S^{\mathrm{UV}}=S^{\mathrm{IR}}-\tilde{S}$ were used, where $\tilde{G}$ and $\tilde{S}$ are defined in (10) and (20).

Then, the divergent double-integrals (44), (54) are canceled in (69) and (70), and the remaining terms are UV finite. With account of spectral and split representations of $G^{\mathrm{IR}}$ and
$\tilde{G}$ [Eqs. (8)-(10)] and of $S^{\mathrm{IR}}$ and $\tilde{S}$ [(19) and (20)], it is seen that the contribution of every of three terms of both $\tilde{\Pi}(71)$, (72) to the expressions of the one-loop self-energy correlators (69), (70) includes corresponding harmonic bubbles (49) and (65). Thus, finally, for UV-finite bubbles (69), (70) defined according to prescription (68), we obtain 


$$
\begin{aligned}
\tilde{\mathcal{M}}_{\Delta_{\phi} \mid \Delta_{\psi} \Delta_{\chi}}^{2 \mathrm{pt}\left(\frac{11}{22}\right)}\left(\vec{x}_{1}, \vec{x}_{2}\right)= & \frac{C_{\Delta_{\phi}}}{P_{12}^{\Delta_{\phi}}} \frac{g_{R}^{2} \operatorname{dim} \gamma}{16 \pi^{d} F^{(0)}\left(\Delta_{\phi}\right)}\left[\mathbf{R}\left(\Delta_{\psi}, \Delta_{\chi} ; \Delta_{\phi}\right)\right. \\
& \left.-\int_{-\infty}^{+\infty} \frac{i d c}{2 \pi} \frac{\mathbf{R}\left(\frac{d}{2}+i c, \Delta_{\chi} ; \Delta_{\phi}\right)}{\left[c+i\left(\Delta_{\psi}-\frac{d}{2}\right)\right]}-\int_{-\infty}^{+\infty} \frac{i d c}{2 \pi} \frac{\mathbf{R}\left(\Delta_{\psi}, \frac{d}{2}+i c ; \Delta_{\phi}\right)}{\left[c+i\left(\Delta_{\chi}-\frac{d}{2}\right)\right]}\right]
\end{aligned}
$$

and

$$
\begin{aligned}
\tilde{\mathcal{M}}_{\Delta_{\psi} \mid \Delta_{\chi}, \Delta_{\phi}}^{2 \mathrm{pt}\left(\frac{1}{2} \frac{1}{2} 0\right)}\left(\vec{x}_{1}, \vec{x}_{2}\right)= & \frac{\hat{C}_{\Delta_{\psi}} \vec{\gamma}\left(\vec{x}_{1}-\vec{x}_{2}\right) \Pi_{-}}{P_{12}^{\Delta_{\psi}+\frac{1}{2}}} \frac{g_{R}^{2}\left(1+\frac{3}{d}\right)}{32 \pi^{d} F^{\left(\frac{1}{2}\right)}\left(\Delta_{\psi}\right)}\left[\mathbf{R}\left(\Delta_{\psi}, \Delta_{\chi} ; \Delta_{\phi}\right)\right. \\
& \left.-\int_{-\infty}^{+\infty} \frac{i d c}{2 \pi} \frac{\mathbf{R}\left(\Delta_{\psi}, \frac{d}{2}+i c ; \Delta_{\phi}\right)}{\left[c+i\left(\Delta_{\chi}-\frac{d}{2}\right)\right]}-\int_{-\infty}^{+\infty} \frac{i c d c \mathbf{R}\left(\Delta_{\psi}, \Delta_{\chi} ; \frac{d}{2}+i c\right)}{2 \pi} \frac{\left[c^{2}+\left(\Delta_{\phi}-\frac{d}{2}\right)^{2}\right]}{\left[c^{2}\right.}\right]
\end{aligned}
$$

where $\mathbf{R}, F^{(0)}$, and $F^{\left(\frac{1}{2}\right)}$ are given in (51)-(52), (50), and (66) correspondingly.

As noted above, function $\mathbf{R}$ for even $d$ may be expressed in terms of elementary functions. In the next section, bubbles (73), (74) will be written explicitly in the $d=4$ Yukawa model.

\section{UV-FINITE BUBBLES IN $S U(N)$ YUKAWA MODEL WITH CONFORMAL SCALAR FIELD IN $d=4$}

Consider bulk Yukawa interaction of $N$ copies of spin-1/2 fields $\psi(Z)$ of mass $m$ with the conformal invariant scalar field $\phi(Z)$ on $\mathrm{AdS}_{5}$ :

$$
L_{\text {int }}=g \phi(Z) \Sigma_{k} \bar{\psi}_{k}(Z) \psi_{k}(Z)
$$

Conformal invariance of $\phi(Z)$ on $\mathrm{AdS}_{d+1}$ means that the equation for the scalar field includes curvature term $\xi_{c} \phi^{2} R_{d+1}$ with the coefficient $\xi_{c}=(d-1) / 4 d$ and that in this case order parameter $\nu=\Delta_{\phi}-d / 2=1 / 2$ in any dimension. Thus, in this model, $m_{\psi}=m_{\chi}=m, \nu=1 / 2$, and it follows for the universal function $\mathbf{R}$ (52) in case $d=4$ that

$$
\mathbf{R}\left(2+m, 2+m ; 2+\frac{1}{2}\right)=\frac{\pi}{4} \frac{\cos ^{2} \pi m}{\cos 2 \pi m}\left(m^{2}-\frac{1}{16}\right)\left(m^{2}-\frac{9}{16}\right) \equiv \tilde{\mathbf{R}}(m) .
$$

Correspondingly, for $\mathbf{R}$ that enter the spectral integrals in the final expressions for bubbles (73), (74), we obtain from (52)

$$
\begin{aligned}
& \mathbf{R}_{(d=4)}\left(2+i c, 2+m ; 2+\frac{1}{2}\right)= \mathbf{R}_{(d=4)}\left(2+m, 2+i c ; 2+\frac{1}{2}\right) \\
&=-\frac{\pi}{8} \frac{\cos \pi m \cosh \pi c}{\cosh 2 \pi c+\cos 2 \pi m}\left[\left(m-\frac{1}{2}\right)^{2}+c^{2}\right]\left[\left(m+\frac{1}{2}\right)^{2}+c^{2}\right] \\
& \cdot\left(\frac{9}{4}-m^{2}+c^{2}-2 i m c\right), \\
& \mathbf{R}_{(d=4)}(2+m, 2+m ; 2+i c)=-\frac{i \pi c^{2} \sinh \pi c \cos ^{2} \pi m}{(\cosh \pi c-1)(\cosh \pi c+\cos 2 \pi m)} \cdot\left[\left(m-\frac{1}{2}\right)^{2}+\frac{c^{2}}{4}\right]\left[\left(m+\frac{1}{2}\right)^{2}+\frac{c^{2}}{4}\right] .
\end{aligned}
$$

Despite the complex nature of (77) and (78) and of the integrands in spectral integrals in (73) and (74), replacing their integration over $c$ from $-\infty$ to $+\infty$ with integration from 0 to $+\infty$ will obviously give the real rhs of (73) and (74).

Having in mind that in the Yukawa model under consideration $\left(d=4, \Delta_{\psi}=\Delta_{\chi}=2+m, \Delta_{\phi}=2+1 / 2\right)$ we have $\operatorname{dim} \gamma=4$ and [see (50) and (66)]

$$
F^{(0)}\left(\Delta_{\phi}=5 / 2\right)=-\frac{3}{16} ; \quad F^{\left(\frac{1}{2}\right)}\left(\Delta_{\psi}=m+2\right)=\left(m^{2}-\frac{1}{4}\right)\left(m^{2}-\frac{9}{4}\right),
$$


substitution of (76)-(78) in (73) and (74) gives final expressions for these one-loop conformal correlators (two spinors and spinor-scalar bubbles),

$$
\begin{aligned}
\tilde{\mathcal{M}}_{\Delta_{\phi}=5 / 2 \mid 2+m, 2+m}^{2 \mathrm{pt}\left(0 \mid \frac{12}{22}\right)(d=4)}\left(\vec{x}_{1}, \vec{x}_{2}\right) & =\frac{C_{5 / 2}}{P_{12}^{5 / 2}} g_{R}^{2} N \Phi^{(\phi)}(m), \\
\Phi^{(\phi)}(m) & =\frac{4}{3 \pi^{4}}\left[-\tilde{\mathbf{R}}(m)-\frac{1}{4} m \cos \pi m I^{(1)}(m)\right]
\end{aligned}
$$

[there is the multiplier $N$ on the rhs because in (75) scalar field $\phi(Z)$ interacts with every spinor field $\psi_{k}(Z)$ ], and

$$
\begin{aligned}
\tilde{\mathcal{M}}_{2+m \mid 2+m, 5 / 2}^{2 \mathrm{pt}\left(\frac{1}{2} \frac{1}{2}\right)(d=4)}\left(\vec{x}_{1}, \vec{x}_{2}\right) & =\frac{\hat{C}_{2+m} \vec{\gamma}\left(\vec{x}_{1}-\vec{x}_{2}\right) \Pi_{-}}{P_{12}^{2+m+\frac{1}{2}}} g_{R}^{2} \Phi^{(\psi)}(m), \\
\Phi^{(\psi)}(m) & =\frac{\left(1+\frac{3}{4}\right)}{32 \pi^{4}} \frac{\left[\tilde{\mathbf{R}}(m)+\frac{1}{8} m \cos \pi m I^{(1)}(m)-\cos ^{2} \pi m I^{(2)}(m)\right]}{\left(m^{2}-\frac{1}{4}\right)\left(m^{2}-\frac{9}{4}\right)},
\end{aligned}
$$

where $\tilde{\mathbf{R}}(m)$ [see (76)], and

$$
\begin{gathered}
I^{(1)}(m)=\int_{0}^{+\infty} \frac{d c \cosh \pi c\left(\frac{9}{4}-m^{2}+3 c^{2}\right)\left[\left(m-\frac{1}{2}\right)^{2}+c^{2}\right]\left[\left(m+\frac{1}{2}\right)^{2}+c^{2}\right]}{\left(c^{2}+m^{2}\right)(\cosh 2 \pi c+\cos 2 \pi m)}, \\
I^{(2)}(m)=\int_{0}^{+\infty} \frac{d c c^{3} \sinh \pi c\left[\left(m-\frac{1}{2}\right)^{2}+\frac{c^{2}}{4}\right]\left[\left(m+\frac{1}{2}\right)^{2}+\frac{c^{2}}{4}\right]}{\left(c^{2}+\frac{1}{4}\right)(\cosh \pi c-1)(\cosh \pi c+\cos 2 \pi m)}
\end{gathered}
$$

are well-defined convergent definite integrals.

Surely transparent expressions similar to (76)-(82), although somewhat more lengthy, may be put down for the model (75) in case $d=4$ when the conformal dimension of scalar field $\Delta_{\phi}=2+\nu$ is arbitrary.

\section{OLD CONFORMAL BOOTSTRAP IN THE AdS/CFT CONTEXT: SPECTRAL EQUATION FOR BULK SPINOR MASS IN YUKAWA MODEL OF SEC. VI}

Expressions for UV-finite quantum one-loop spinorscalar contributions (bubbles) to scalar (73) [or (79) in the Yukawa model (75)] and spinor (74) [Eq. (80) in the model in (75)] boundary-boundary conformal correlators may be used in different ways:

(i) for the calculation of anomalous dimensions generated by the scalar-spinor bulk loops like was done in Ref. [40] for bubbles built of the fields of integer spin with the use of logarithmic terms in the dimensional regularization of conformal divergent integrals like (48) or (64).

(ii) for presenting decomposition of the bubbles built of two spinors or of a spinor and scalar in an infinite series of residues in poles of integrands in spectral integrals in (73) and (74); the locations and residues of these poles are evident from expressions (51) and (52) [or (77) and (78) in the model in (75)] for coefficient $\mathbf{R}$ determining harmonic bubbles (49),
(65); detailed analysis of poles of harmonic bubbles formed by the fields of integer spin was presented in Ref. [40], etc.

Here, we pay attention that expressions obtained above permit formulating spectral equations for the bulk masses of spinors in frames of the old conformal bootstrap in the AdS/CFT context $[12,13]$. The simplest old conformal bootstrap equation for the Green's function $G(X, Y)$ traditionally written in planar approximation looks like [14-22]

$$
\begin{aligned}
G\left(X_{1}, X_{2}\right)= & g^{2} \iint G\left(X_{1}, X\right) G(X, Y) G(X, Y) \\
& \times G\left(Y, X_{2}\right) d X d Y
\end{aligned}
$$

(triple interaction is supposed, and $g$ is the coupling constant). Equating of the exact Green's functions to the one-loop quantum contribution built of the same exact Green's functions is the main postulate of the old conformal bootstrap. This type of bootstrap equation was also used recently for the calculation of spectra of conformal dimensions in one-dimensional Sachdev-Ye-Kitaev and in $d$-dimensional field theory models [51].

Equation (83) is just a conventional Schwinger-Dyson equation in which terms associated with bare Lagrangian are omitted. This may be called the zero-Lagrangian approach applied by Sakharov in his quantum-induced theory of gravity [52] (the attempt to follow this method in the AdS context was made in $[27,28]$, in which UV finite induced gravitational and gauge coupling constants were 
calculated). The similar "bootstrap" equations with zero "bare mass" terms are widely used in different approaches to dynamical symmetry breaking and mass generation pioneered in Refs. [53,54], etc.

In the AdS/CFT context, it is assumed in Refs. [12,13] that $X_{1,2}, X$, and $Y$ in (83) are the bulk coordinates in $\operatorname{AdS}_{d+1}$ and $X_{1,2}$ are sent to the horizon. This procedure ${ }^{1}$ transforms the lhs of (83) into a conformal correlator of the boundary conformal theory [Eq. (7) for the scalar and (13) for the spinor], whereas $G\left(X_{1}, X\right)$ and $G\left(Y, X_{2}\right)$ on the rhs become the corresponding bulk-to-boundary propagators. Thus, the rhs of (83) becomes the quantum one-loop correlator (bubble). In this way, different bootstrap equations may be obtained depending on the choice of boundary conditions for $G\left(X_{1}, X\right)$ and $G\left(Y, X_{2}\right)$ in (83); in what follows, I use "both IR" boundary conditions for these Green's functions (the "both UV" option will give the same spectral equations, as was shown in Ref. [13], in which the model of interacting scalar fields was considered).

Applying old bootstrap equation (83) (where $X_{1,2}$ are sent to the horizon) to scalar and spinor fields and defining the UV-finite bubble on the rhs of (83) according to the "double-trace subtraction" postulate of Sec. V, we obtain from (83) with account of (7) and (13) for the lhs and (73) and (74) for the rhs

$$
\begin{aligned}
\frac{C_{\Delta_{\phi}}}{P_{12}^{\Delta_{\phi}}} & =\tilde{\mathcal{M}}_{\Delta_{\phi} \mid \Delta_{\psi} \Delta_{\chi}}^{2 \mathrm{pt}\left(0 \mid \frac{11}{22}\right)}\left(\vec{x}_{1}, \vec{x}_{2}\right) ; \\
\frac{\hat{C}_{\Delta_{\psi}} \vec{\gamma}\left(\vec{x}_{1}-\vec{x}_{2}\right) \Pi_{-}}{P_{12}^{\Delta_{\psi}+\frac{1}{2}}} & =\tilde{\mathcal{M}}_{\Delta_{\psi} \mid \Delta_{\chi}, \Delta_{\phi}}^{2 \mathrm{pt}\left(\frac{1}{2} \frac{1}{2} 0\right)}\left(\vec{x}_{1}, \vec{x}_{2}\right) .
\end{aligned}
$$

Reducing the similar space-time and spinor dependence of the lhs and rhs of Eqs. (84), the following old bootstrap equations are obtained from (84) for the particular case of the model in (75) when bubbles are given by (79) and (80):

$$
\begin{gathered}
1=g_{R}^{2} N \Phi^{(\phi)}(m), \\
1=g_{R}^{2} \Phi^{(\psi)}(m) .
\end{gathered}
$$

After elimination here of $g_{R}^{2}$, the interesting spectral equation for spinor bulk mass $m$ is obtained:

$$
N \Phi^{(\phi)}(m)=\Phi^{(\psi)}(m)
$$

In Ref. [13], a similar spectral equation for conformal dimensions in the $O(N)$ symmetric model of $N$ scalar fields interacting with the conformal invariant HubbardStratonovich field is derived, and its roots obeying the unitarity bound demand are found: three roots for every $N=1,2,3,4$.

\footnotetext{
${ }^{1}$ I am grateful to Ruslan Metsaev for this observation.
}

The spectral equation (87) for every $N$ possesses positive and negative roots; however, only one of them belongs to the physically interesting interval (3), and its value $m \cong$ 0.87 weakly depends on $N$. According to (2), this value of bulk fermion mass gives the mass of physical fermion excitation $p_{0} \cong 0.05 \mathrm{MeV}$.

The interesting task would be to consider the spontaneous breakdown of $S U(N)$ symmetry in the model in (75). This means that every spinor field $\psi_{k}(Z)$ is supposed to have its own bulk mass $m_{k}$ and in expression (79) for the 2-spinor bubble of scalar field $\phi(Z)$ the multiplication by $N$ should be replaced by the sum over $k, N \Phi^{(\phi)}(m) \rightarrow$ $\Sigma_{k} \Phi^{(\phi)}\left(m_{k}\right)$, whereas expression (80) should be valid for every $m_{k}$. Then, spectral equation (87) takes a form of system of $N$ equations $(k=1,2 \ldots N)$ for $N$ unknowns $m_{k}$ $\left[\Phi^{(\phi, \psi)}(m)\right.$; see (79) and (80)]:

$$
\Sigma_{i} \Phi^{(\phi)}\left(m_{i}\right)=\Phi^{(\psi)}\left(m_{k}\right) .
$$

The physically intriguing goal is the search for roots $m_{1} \ldots m_{N}$ of system (88) that belong to interval (3) with the hope to get, from Eq. (2), for example, the observed masses of spin-1/2 flavors.

Surely the assumption of conformal invariance of the scalar field in the Yukawa model (75) is just a demonstrative one. It is not difficult to put down the system of spectral equations similar to (88) for arbitrary conformal dimension of scalar field $\Delta_{\phi}$ and to get the dependence of roots $m_{k}$ on $\Delta_{\phi}$, with the hope that for some $\Delta_{\phi}$ and $N$ the physically interesting combinations of $N$ roots $m_{1} \ldots m_{N}$ will be obtained.

\section{CONCLUSION}

The results of this paper are threefold; in every direction, there is possible further development.

First, the transparent expressions are obtained in physical $\mathrm{AdS}_{d+1}$ space for spinor-scalar vertices and for two-point one-loop quantum conformal correlators (bubbles) formed with participation of spin- $1 / 2$ bulk fields. If the scalar field bubble formed with two spinors was calculated earlier in the formalism of embedding space [39], the result for the loop formed by a spinor and a scalar has not been met in the literature, as to my knowledge. The fantastic simplicity of this result obtained through lengthy calculations gives rise to the hunch that perhaps there is a simpler way to achieve it. The option of Yukawa bulk interaction $\phi(Z) \bar{\psi}(Z) \psi(Z)$ was considered above, and immediate generalization of the approach of the paper may be the calculation of vertices and bubbles in the cases of pseudoscalar $\pi(Z) \bar{\psi}(Z) \gamma^{5} \psi(Z)$ or vector $V_{\mu}(Z) \bar{\psi}(Z) \gamma^{\mu} \psi(Z)$ interactions, the bulk QED as a special option.

Second, the novel tool of the elimination of the UV divergence of bubble Witten diagrams is proposed and actually repeats the approach used earlier in [23-30] in calculations of Witten tadpoles. It would be interesting to 
check if the difference of the UV and IR Witten triangle and other diagrams proves to be UV finite as happened in Sec. V for bubble diagrams.

Third, the expressions for UV-finite spinor-scalar bubbles received in the paper permit applying them in the old conformal bootstrap equations aimed at the calculation of spectra of bulk spinor masses. The task for the future may be the calculation of roots of spectral equation (87) or of system of Eqs. (88) and of similar equations received in models with pseudoscalar or vector interactions of spin- $1 / 2$ fields. As was outlined in the Introduction, the knowledge of bulk spinor masses may be a way to the solution of the longstanding fermion mass hierarchy, or flavors mass hierarchy, problem.

\section{ACKNOWLEDGMENTS}

The author is grateful to Ruslan Metsaev, Mitsuhiro Nishida, Kotaro Tamaoka, Dmitry Nesterov, Alexander Smirnov, Arkady Tseytlin, and Mikhail Vasiliev for stimulating comments and assistance and to participants of the seminar in the Theoretical Physics Department of the P. N. Lebedev Physical Institute for useful questions.

\section{APPENDIX: DERIVATION OF FORMULAS (24) AND (29)}

\section{Derivation of $D^{(1)}(24)$}

Calculation of bulk integral $D_{\gamma_{1} \gamma_{2} \gamma_{3}}^{(\alpha)}\left(\vec{x}_{1}, \vec{x}_{2}, \vec{x}_{3}\right)(21)$ is performed in an ordinary way with the introduction of Schwinger parameters-see, for example, Sec. 3.1 and Appendix A in Ref. [49]. The representation of $D^{(\alpha)}$ as integral over Schwinger parameters differs from the wellknown one for $D^{(0)}$ only in the argument of the first Gamma function and in the additional factor $\left(\Sigma_{i} t_{i}\right)^{\alpha}$ in the integrand $(i=1,2,3)$,

$$
\begin{aligned}
D_{\gamma_{1} \gamma_{2} \gamma_{3}}^{(\alpha)}\left(\vec{x}_{1}, \vec{x}_{2}, \vec{x}_{3}\right)= & \pi^{\frac{d}{2}} \frac{\Gamma\left(\frac{\Sigma_{i} \gamma_{i}-d-\alpha^{-1}}{2}\right)}{\Gamma\left(\gamma_{1}\right) \Gamma\left(\gamma_{2}\right) \Gamma\left(\gamma_{3}\right)} \int \Pi_{i}\left(\frac{d t_{i}}{t_{i}} t_{i}^{\gamma_{i}}\right) \\
& \times\left(\Sigma_{i} t_{i}\right)^{\alpha} e^{-Q^{2}},
\end{aligned}
$$

where

$$
Q^{2}=t_{1} t_{2} P_{12}+t_{1} t_{3} P_{13}+t_{2} t_{3} P_{23}
$$

for $P_{i j}$, see (6).

Thus, in case $\alpha=1$, the standard change in (A1) of the integration variables

$$
t_{1}=\sqrt{\frac{m_{2} m_{3}}{m_{1}}} \quad t_{2}=\sqrt{\frac{m_{1} m_{3}}{m_{2}}} \quad t_{3}=\sqrt{\frac{m_{1} m_{2}}{m_{3}}}
$$

and simple algebra immediately give for $D^{(1)}$ expression (24).

\section{Derivation of $\boldsymbol{R}^{(1)}$ (29)}

Derivation of $R_{\beta_{1} \beta_{2} \beta_{3}}^{(1)}\left(\vec{x}_{1}, \vec{x}_{2}, \vec{x}_{3}\right)$ is again performed in an ordinary way with the introduction of Schwinger parameters, that gives

$R_{\beta_{1} \beta_{2} \beta_{3}}^{(1)}\left(\vec{x}_{1}, \vec{x}_{2}, \vec{x}_{3}\right)=2 \pi^{\frac{d}{2}} \int \Pi_{i}\left(\frac{d t_{i}}{t_{i}} \frac{t_{i}^{\beta_{i}}}{\Gamma\left(\beta_{i}\right)}\right)\left(\Sigma_{i} t_{i}\right)^{\Sigma_{i} \beta_{i}-d} e^{-Q^{2}}$

for $Q^{2}$, see above in Appendix A 1 . For $\Sigma_{i} \beta_{i}=d+1$, the integral in (A2) coincides with the integral in (A1) at $\alpha=1$, and (29) follows from (A2) in the same way in which (24) was obtained from (A1).
[1] C. E. Diaz, S. F. Mantilla, and R. Martinez, Fermion mass hierarchy from nonuniversal abelian extensions of the Standard Model, Phys. Rev. D 98, 015038 (2018).

[2] G. Abbas, Solving the fermionic mass hierarchy of the standard model, Int. J. Mod. Phys. A 34, 1950104 (2019).

[3] S. Weinberg, Model physicist, Interview to Matthew Chalmers, CERN Courier, 2017, http://cerncourier.com/ cws/article/cern/70138.

[4] L. Randall and R. Sundrum, A Large Mass Hierarchy from a Small Extra Dimension, Phys. Rev. Lett. 83, 3370 (1999).

[5] T. Gherghetta and A. Pomarol, Bulk fields and supersymmetry in a slice of AdS, Nucl. Phys. B586, 141 (2000).

[6] S. J. Huber and Q. Shafi, Fermion masses, mixings and proton decay in a Randall-Sundrum model, Phys. Lett. B 498, 256 (2001).
[7] T. Gherghetta, K. Kadota, and M. Yamaguchi, Warped leptogenesis with Dirac neutrino masses, Phys. Rev. D 76, 023516 (2017).

[8] B. L. Altshuler, Electron neutrino mass scale in spectrum of Dirac equation with the 5-form flux term on the $\operatorname{AdS}(5) \times S$ (5) background, J. High Energy Phys. 08 (2009) 091.

[9] P. R. Archer, The fermion mass hierarchy in models with warped extra dimensions and a bulk higgs, J. High Energy Phys. 09 (2012) 095.

[10] Y. Grossman and M. Neubert, Neutrino masses and mixings in non-factorizable geometry, Phys. Lett. B 474, 361 (2000).

[11] T. Gherghetta and A. Pomarol, A warped supersymmetric standard model, Nucl. Phys. B602, 3 (2001).

[12] B. L. Altshuler, "Old" conformal bootstrap in the AdS/CFT context, arXiv:1810.01105; Old conformal bootstrap on 
AdS: O(N) symmetric scalar model, Int. J. Mod. Phys. A35, 2050001 (2020).

[13] B. L. Altshuler, UV-finite "old" conformal bootstrap on AdS: Scalar case, J. High Energy Phys. 01 (2020) 137.

[14] A. M. Polyakov, Microscopic description of critical phenomena, Zh. Exp. Theor. Fiz. 55, 1026 (1968) [Sov. Phys. JETP 28, 533 (1969)].

[15] A. A. Migdal, A diagram technique near the curie point and the second order phase transition in a bose liquid, Zh. Exp. Theor. Fiz. 55, 1964 (1968) [Sov. Phys. JETP 28, 1036 (1969)]; Conformal invariance and bootstrap, Phys. Lett. 37B, 386 (1971); Ancient History of CFT, http://migdal100.itp.ac.ru/ proceedings_files/MigdalAA_AncientHistoryOfCFT.pdf.

[16] G. Parisi and L. Peliti, Calculation of critical indices, Lett. Nuovo Cimento 2, 627 (1971); M. d'Eramo, G. Parisi, and L. Peliti, Theoretical predictions for critical exponents at the $\lambda$-point of bose liquids, Lett. Nuovo Cimento 2, 878 (1971); G. Parisi, On self-consistency conditions in conformal covariant field theory, Lett. Nuovo Cimento 4, 777 (1972).

[17] E. S. Fradkin and M. Ya. Palchik, Conformal Quantum Field Theory in D Dimensions (Kluwer, Dordrecht, Netherlands, 1996), p. 461; E. S. Fradkin and M. Ya. Palchik, Recent developments in conformal-invariant quantum field theory, Phys. Rep. 44C, 249 (1978).

[18] G. Mack and K. Symanzik, Currents, stress tensor and generalized unitarity in conformal invariant quantum field theory, Commun. Math. Phys. 27, 247 (1972).

[19] G. Mack and T. Todorov, Conformal-invariant Green functions without ultraviolet divergences, Phys. Rev. D 8, 1764 (1973).

[20] V. K. Dobrev, V. B. Petkova, S. G. Petrova, and I. T. Todorov, Dynamical derivation of vacuum operator product expansion in Euclidean conformal quantum field theory, Phys. Rev. D 13, 887 (1976).

[21] V. K. Dobrev, G. Mack, V. B. Petkova, S. G. Petrova, and I. T. Todorov, Harmonic Analysis on the n-Dimensional Lorenz, Group and its Applications to Conformal Quantum Field Theory, Lecture Notes in Physics, (Springer Verlag, Berlin-Heidelberg-New York, 1977), Vol. 63, p. 280.

[22] D. Grensing and G. Grensing, Critical indices and the conformal invariant bootstrap method, Phys. Rev. D 18, 2890 (1978).

[23] S. S. Gubser and I. Mitra, Double-trace operators and one loop vacuum energy in AdS/CFT, Phys. Rev. D 67, 064018 (2003).

[24] T. Hartman and L. Rastelli, Double-trace deformations, mixed boundary conditions and functional determinants in AdS/CFT, J. High Energy Phys. 01 (2008) 019.

[25] D. E. Diaz and H. Dorn, Partition functions and double-trace deformations in AdS/CFT, J. High Energy Phys. 05 (2007) 046.

[26] A. A. Saharian, Wightman function and Casimir densities on AdS bulk with application to the Randall-Sundrum braneworld, Nucl. Phys. B712, 196 (2005).

[27] B. L. Altshuler, Sakharov's induced gravity on the AdS background. SM scale as inverse mass parameter of Schwinger-DeWitt expansion, Phys. Rev. D 92, 065007 (2015).

[28] B. L. Altshuler, Simple way to calculate UV-finite one-loop quantum energy in Randall-Sundrum model, Phys. Rev. D
95, 086001 (2017); Scalar field on AdS: Quantum one loop "in one line," Report at the Ginzburg Centennial Conference, Lebedev Institute, Moscow, 2017 arXiv: 1706.06286 .

[29] A. Allais, Double-trace deformations, holography and the $c$-conjecture, J. High Energy Phys. 11 (2010) 040.

[30] R. Aros and D. E. Diaz, Determinant and Weyl anomaly of Dirac operator: A holographic derivation, J. Phys. A $\mathbf{4 5}$, 125401 (2012).

[31] M. Henningson and K. Sfetsos, Spinors and the AdS/CFT correspondence, Phys. Lett. B 431, 63 (1998).

[32] W. Mueck and K. S. Viswanathan, Conformal field theory correlators from classical field theory on anti-de Sitter space. 2. Vector and spinor fields, Phys. Rev. D 58, 106006 (1998).

[33] M. Henneaux, Boundary terms in the AdS/CFT correspondence for spinor fields, arXiv:hep-th/9902137.

[34] T. Kawano and K. Okuyama, Spinor exchange in $\operatorname{AdS}(d+1)$, Nucl. Phys. B565, 427 (2000).

[35] R. C. Rashkov, Regular and irregular boundary conditions in AdS/CFT correspondence for spinor field, Phys. Lett. 466, 190 (1999).

[36] Y. Hikida and T. Wada, Marginal deformations of $3 \mathrm{~d}$ supersymmetric $\mathrm{U}(\mathrm{N})$ model and broken higher spin symmetry, J. High Energy Phys. 03 (2017) 047.

[37] H. Isono, On conformal correlators and blocks with spinors in general dimensions, Phys. Rev. D 96, 065011 (2017).

[38] M. Nishida and K. Tamaoka, Fermions in geodesic witten diagrams, J. High Energy Phys. 07 (2018) 149.

[39] D. Carmi, L. Di Pietro, and S. Komatsu, A study of quantum field theories in AdS at finite coupling, J. High Energy Phys. 01 (2019) 200.

[40] S. Giombi, C. Sleight, and M. Taronna, Spinning AdS Loop Diagrams: Two Point Functions, J. High Energy Phys. 06 (2018) 030.

[41] I. R. Klebanov and E. Witten, AdS/CFT correspondence and symmetry breaking, Nucl. Phys. B556, 89 (1999).

[42] S. Giombi, V. Kirilin, and E. Perlmutter, Double-trace deformations of conformal correlations, J. High Energy Phys. 02 (2018) 175.

[43] A. O. Barvinsky and D. V. Nesterov, Schwinger-DeWitt technique for quantum effective action in brane induced gravity models, Phys. Rev. D 81, 085018 (2010).

[44] J. Penedones, Writing CFT correlation functions as AdS scattering amplitudes, J. High Energy Phys. 03 (2011) 025.

[45] A. L. Fitzpatrick, J. Kaplan, J. Penedones, S. Raju, and B. C. van Rees, A natural language for AdS/CFT correlators, J. High Energy Phys. 11 (2011) 95.

[46] M. S. Costa, V. Goncalves, and J. Penedones, Spinning AdS propagators, J. High Energy Phys. 09 (2014) 064.

[47] X. Bekaert, J. Erdmenger, D. Ponomarev, and S. Sleight, Towards holographic higher-spin interactions: Four-point functions and higher-spin exchange, J. High Energy Phys. 03 (2015) 170.

[48] D. Z. Freedman, S. D. Mathur, A. Matusis, and L. Rastelli, Correlation functions in the $\mathrm{CFT}_{d} / \mathrm{AdS}_{d+1}$ correspondence, Nucl. Phys. B546, 96 (1999). 
[49] M. F. Paulos, Towards Feynman rules for Mellin amplitudes in AdS/CFT, J. High Energy Phys. 10 (2011) 74.

[50] K. Symanzik, On calculations in conformal invariant field theories, Lett. Nuovo Cimento 3, 734 (1972).

[51] I. R. Klebanov and G. Tarnopolsky, Uncolored random tensors, melon diagrams, and the SYK models, Phys. Rev. D 95, 046004 (2017).

[52] A. D. Sakharov, Vacuum Quantum Fluctuations In Curved Space And The Theory Of Gravitation, Dokl. Akad. Nauk
Ser. Fiz. 177, 70 (1968) [Sov. Phys. Dokl. 12, 1040 (1968)]; reprinted in Gen. Relativ. Gravit. 32, 365 (2000).

[53] Y. Nambu and G. Jona-Lasinio, Dynamical model of elementary particles based on an analogy with superconductivity, Phys. Rev. 122, 345 (1961).

[54] S. Coleman and E. J. Weinberg, Radiative corrections as the origin of spontaneous symmetry breaking, Phys. Rev. D 8, 1888 (1973). 